\title{
Towards a hyperintensional theory of intrinsicality
}

\author{
Ralf M. Bader \\ Merton College, University of Oxford
}

(forthcoming in The Journal of Philosophy)

\begin{abstract}
Aвsтract: The distinction between intrinsic and extrinsic properties is an elusive distinction that has resisted precise formulation. Some of the most promising accounts of this distinction take the form of combinatorial or duplication analyses. The former try to capture the patterns of independence amongst intrinsic and extrinsic properties in terms of logical, modal, mereological and spatiotemporal notions. The latter attempt to identify intrinsic properties as properties that never differ amongst duplicates. This paper argues in favour of a hyperintensional analysis of intrinsicality that appeals to 'in virtue of' claims. It will be shown that accounts of intrinsicality that appeal to combinatorial and duplication principles do not yield satisfactory results, even when they are supplemented with a notion of 'naturalness'. We need to appeal to 'in virtue of' claims rather than to 'naturalness' in order (i) to allow for cases whereby a property is possessed both intrinsically and extrinsically, (ii) to adequately classify modal properties when these are given a counterparttheoretic analysis, and (iii) to retain the idea that the set of intrinsic properties and the set of pure extrinsic properties are closed under Boolean operations. Moreover, the paper will argue in favour of treating the intrinsically/extrinsically distinction as more basic than the intrinsic/extrinsic distinction and explaining the latter in terms of the former.
\end{abstract}




\section{Introduction}

The distinction between intrinsic and extrinsic properties is an elusive distinction that has resisted precise formulation. Analyses of this distinction are guided by two platitudes about intrinsicality:

I. intrinsic properties are had solely in virtue of how a thing itself is and not in virtue of how it is related to other things

2. the instantiation of intrinsic properties is independent of how the rest of the world is (intrinsically)

The task of the metaphysician consists in giving an analysis of the distinction between intrinsic and extrinsic properties that captures these platitudes. Two promising strategies that are widely accepted and discussed appeal to combinatorial principles and duplication principles, whereby in each case the notion of naturalness is brought into the analysis.

This paper argues in favour of a hyperintensional analysis of intrinsicality that appeals to 'in virtue of' claims. Accounts that are extensional in nature will be shown to be inadequate even when they are supplemented by considerations of naturalness. Such accounts individuate properties in terms of their (actual as well as possible) instances ${ }^{\mathrm{I}}$ and thereby commit themselves to sameness of extension implying sameness of property. Moreover, they do not recognise different ways in which properties can be instantiated. This renders extensional approaches problematic since it implies that they mistakenly make being $\mathrm{F}$ intrinsically and being $\mathrm{F}$ extrinsically incompatible, thereby precluding the possibility of a property being possessed both intrinsically and extrinsically at the same time. This inadequacy can be illustrated by cases involving objects that have both intrinsic and extrinsic value, i.e. objects that are both intrinsically and extrinsically valuable (section 2). In addition, it will be shown that these accounts are problematic since they cannot adequately deal with extra-world sources of extrinsicness. In particular, they are unable to adequately classify properties that are cross-world dependent yet intra-world independent, such as certain modal properties when these are given a counterpart-theoretic analysis (section 3). Moreover, extensional accounts face difficulties when it comes to dealing with necessary and impossible properties. In particular, they cannot retain the idea that the set of intrinsic properties and the set of pure extrinsic properties are closed under Boolean operations (section 4). In general, we will see that the problem with all these extensional accounts is that they only assess whether a property is had and not how it is had. They thereby ignore important hyperintensional differences in the ways in which properties can be had. These shortcomings cannot be remedied by appealing to

\footnotetext{
I'Extensional' is here understood in the context of a possibilist domain of quantification and is accordingly contrasted with 'hyperintensional' rather than with 'intensional'. Quantifiers in this paper are taken to range over possibilia unless otherwise indicated.
} 
naturalness. Instead, we need to develop a hyperintensional theory of intrinsicality that allows for a more fine-grained understanding of how properties are instantiated and individuated. On the one hand, such a theory enables us to differentiate properties that have the same extension, which is particularly important when it comes to the intrinsicality of necessary properties, as well as when dealing with cases in which absorption principles would naturally seem to fail. On the other hand, it allows us to distinguish different ways in which properties can be had, which, on the one hand, allows us to deal with properties that, though having different extensions than intrinsic properties, have isomorphic modal profiles and, on the other, is crucial when it comes to dealing with cases in which a property is had both intrinsically and extrinsically. A hyperintensional account allows us to move from the extensional question of whether a property is instantiated to the hyperintensional question of how it is instantiated, which is understood in terms of the ground of the instantiation of the property, and thereby makes room for the possibility of one and the same property being instantiated in different ways by the same object at the same time.

Such a hyperintensional account will be provided in the second half of the paper (section 5), where it will be argued that we should construe the local notion of intrinsicality (the intrinsically/extrinsically distinction) as being more basic than the global notion (the intrinsic/extrinsic distinction) and appeal to the former in explicating the latter. ${ }^{2}$ While there have been many attempts to analyse the global notion, the local notion has been unduly neglected, which is problematic insofar as it has significant philosophical importance since it is this notion that is, for instance, at issue when one is concerned with the question whether something is intrinsically or extrinsically valuable. Moreover, it turns out to be the local notion that is the more fundamental one and that needs to be explicated first.

\section{I.I Combinatorial principles}

The combinatorial strategy involves giving extensional analyses and principles of intrinsicality that characterise the patterns of modal independence exhibited by intrinsic properties in terms of logical, modal, mereological and spatio-temporal notions. The first explicit account falling under this strategy was based on Moore's isolation test, as well as on suggestions made by Chisholm, and was developed by Kim (cf. Kim: 1982, pp. 59-60). The isolation principle states that a property $\mathrm{F}$ is an intrinsic property if it can be had by a lonely object, i.e. an object not accompanied by any wholly distinct contingent existent. This account was criticised by Lewis on the basis that it was not able to deal with disjunctive properties, as well

${ }^{2}$ For the distinction between the global and the local notion of intrinsicality cf. Dunn: I990, p. I83 \& Humberstone: I996, p. 206 and pp. 227-228. (The terminology is due to Humberstone.) The former notion is concerned with whether a property is an intrinsic or an extrinsic property. The latter notion is concerned with whether a particular object possesses a property intrinsically or extrinsically. 
as on the basis that negative extrinsic properties, such as the property of loneliness, can be had by isolated objects (cf. Lewis: 1983b). Refined combinatorial accounts which appeal to the notion of independence of accompaniment were then proposed by Langton and Lewis as well as by Zimmerman (cf. Langton and Lewis: 1998 \& Zimmerman: 1997, Appendix A). On these accounts, a property $\mathrm{F}$ is a basic intrinsic property if it is (i) neither disjunctive nor the negation of a disjunction and (ii) independent of accompaniment, which means that there can be a lonely F, a lonely non-F, an accompanied F, and an accompanied non-F. Yet, these accounts were not without problems either and they were in turn replaced by analyses that are holistic in nature, the most sophisticated versions having been developed by Weatherson and Denby (cf. Weatherson: 200 I \& Denby: 2006). Holistic accounts do not provide principles that allow us to identify particular intrinsic properties but rather specify conditions on the set of intrinsic properties, such as Boolean closure conditions.

As is well known, combinatorial accounts are problematic in that they cannot deal with disjunctive mixed extrinsic properties of the form $\left(\left(I_{I} \wedge A\right) \vee\left(I_{2} \wedge\right.\right.$ $L)$ ), where $I_{I}$ and $I_{2}$ are incompatible intrinsic properties while $A$ is the property of being accompanied and L the property of being lonely, such as the property 'being either an accompanied electron or a lonely positron'. 3 Such disjunctive mixed extrinsic properties exhibit the same patterns of modal independence as intrinsic properties and are therefore indistinguishable from them by means of combinatorial tools. ${ }^{4}$ Combinatorial principles are consequently unable to pin down the distinction between intrinsic and extrinsic properties since they do not lead to a unique assignment of properties. As a result, there is a need to supplement combinatorial accounts and appeal to other considerations that allow us to distinguish these two kinds of properties. In order to avoid counter-examples involving disjunctive properties, these accounts are generally supplemented by conditions invoking naturalness. For example, when dealing with a holistic account such as the one proposed by Denby, we need to judge that the pair of properties $<\mathrm{I}_{\mathrm{I}}, \mathrm{I}_{2}>$ is more natural than the pair $<\left(\left(\mathrm{I}_{\mathrm{I}} \wedge \mathrm{A}\right) \vee\left(I_{2} \wedge \mathrm{L}\right)\right),\left(\left(I_{2} \wedge A\right) \vee\right.$ $\left.\left(I_{I} \wedge L\right)\right)>$, in order to bring it about that the members of the former pair rather than those of the latter pair are included in the set of intrinsic properties.

${ }^{3}$ Cf. Parsons: 200I, pp. 22-23, Weatherson: 200I, p. 375 \& Marshall: 2009.

${ }^{4}$ The problem here is in important respects analogous to the problem of grue in that we also have two pairs of properties in that case, namely grue/bleen and green/blue, that are interdefinable and that have isomorphic modal profiles and can accordingly not be differentiated by extensional means. Troublesome disjunctive properties of this kind also generate problems in ethics and choice theory where they threaten to undermine separability principles as well as choice consistency conditions, and where one needs to bring in hyperintensional independence conditions to deal with them (cf. Bader: manuscript-a). 


\section{I.2 Duplication principles}

The duplication strategy attempts to give an account of intrinsicality in terms of properties that are shared by duplicates (cf. Lewis: 1983a, I983b \& 1986). According to the duplication analysis of the global notion of intrinsicality, intrinsic properties are properties that never differ amongst duplicates, while extrinsic properties are properties that do differ amongst duplicates. According to the duplication analysis of the local notion of intrinsicality, $\mathrm{x}$ is $\mathrm{F}$ intrinsically iff all duplicates of $\mathrm{x}$ are $\mathrm{F}^{5}$, while $\mathrm{x}$ is $\mathrm{F}$ extrinsically iff $\mathrm{x}$ is $\mathrm{F}$ and some duplicate of $\mathrm{x}$ fails to be $F$.

An important problem for this way of analysing intrinsicality is that it ends up in a definitional circle. In order to apply the duplication account we need to know what it means for $\mathrm{x}$ and $\mathrm{y}$ to be duplicates. The problem now is that the most plausible answer to this question is that $\mathrm{x}$ and $\mathrm{y}$ are duplicates iff they share all the same intrinsic properties. It thus seems that we end up in a definitional circle since duplicates are understood in terms of intrinsic properties and intrinsic properties are characterised by means of duplicates. As Lewis notes: "We have a tight little family of interdefinables" (Lewis: 1983b, p. 197).

Lewis suggests that we can break this circle by appealing to perfectly natural properties. In particular, rather than defining duplicates as objects that share all the same intrinsic properties, we can define duplicates as objects that have the same perfectly natural properties.

DUPLICATE $\mathrm{x}$ and $\mathrm{y}$ are duplicates iff there is a bijection $\Gamma$ from the parts of $\mathrm{x}$ to the parts of $y$, such that any perfectly natural property $\mathrm{F}$ is instantiated by any part $\mathrm{p}$ of $\mathrm{x}$ iff the image of $\mathrm{p}$ under $\Gamma$ is also $\mathrm{F}$, and such that any n-ary perfectly natural relation $R$ holds amongst parts $\mathrm{p}_{\mathrm{I}} \ldots \mathrm{p}_{\mathrm{n}}$ of $\mathrm{x}$ iff the images of $\mathrm{p}_{\mathrm{I}} \ldots \mathrm{p}_{\mathrm{n}}$ under $\Gamma$ also stand in $\mathrm{R}$.

\section{I.3 Naturalness}

We have seen that the notion of naturalness plays a central role in both approaches. Combinatorial accounts have to resort to naturalness to deal with disjunctive properties, while duplication accounts require the notion of perfectly natural properties to escape the 'tight definitional circle', allowing them to give a specification of what duplicates are without invoking intrinsic properties.

It is worthwhile pointing out that the combinatorial accounts require only judgements of comparative naturalness. One property is judged to be less natural, i.e. more disjunctive, than another. Duplication accounts, however, presuppose that there are perfectly natural properties since duplicates are understood in terms of isomorphisms that preserve perfectly natural properties and relations. This is more problematic given that properties might turn out to stand in some hierarchy

\footnotetext{
${ }^{5}$ Given the reflexivity of the duplication relation, $\mathrm{x}$ is a duplicate of itself.
} 
whereby they get more and more natural as one goes down the hierarchy without there being any perfectly or most natural properties, i.e. the hierarchy lacks a lower bound.

This appeal to naturalness has been criticised on a number of occasions. In particular, it has been objected that this is not a well-understood notion that is explanatorily useful and that there are no clear criteria for assessing the comparative naturalness of different properties - "the problem is a matter of having no clear concept the costs and benefits of which can even begin to be assessed" (Witmer, Butchard and Trogdon: 2005, p. 329; also cf. Yablo: 1999, section II).

In this paper, I will grant for the sake of argument that appealing to considerations of naturalness is legitimate, that there are perfectly natural properties and that judgements of comparative naturalness are unproblematic. Instead of raising doubts about the notion of naturalness or its usefulness in comparing properties, I will show that duplication accounts as well as other extensional accounts supplemented by naturalness are inadequate. There are problems that these accounts face which cannot be resolved by appealing to naturalness. Since the notion of intrinsicality lies at the very core of the Lewisian/Humean project $^{6}$, the fact that it cannot be accounted for by means of resources acceptable to adherents of this approach raises serious concerns about the viability of this project. Unlike external critiques that question the notion of naturalness, the argument put forward in this paper classifies as an internal critique that cannot be accused of begging the question.

Rather than being isolated problem cases, these difficulties turn out to be symptomatic of an underlying inadequacy. The modal independence which combinatorial and duplication accounts track is nothing more than a surface manifestation that does not capture the nature of intrinsic properties. Intrinsic properties exhibit certain patterns of modal independence because they are had in virtue of the way things themselves are and we have to appeal to this 'in virtue of' relation if we are to adequately characterise the notion of intrinsicality. This suggests that an altogether different approach is required for dealing with intrinsicality, which will be developed in the last section. Although combinatorial and duplication principles do not enable us to give an analysis of intrinsicality, they can nonetheless play a limited role in a proper account of intrinsicality insofar as they allow us to specify necessary though not sufficient conditions for a property to be an intrinsic property/to be had intrinsically by $\mathrm{x}$, as well as sufficient though not necessary conditions for a property to be an extrinsic property/to be had extrinsically by $\mathrm{x}$ (cf. section 5.2).

\footnotetext{
${ }^{6}$ For instance, intrinsicness plays a central role insofar as (i) the spatio-temporal distribution of qualitative intrinsic properties characterises the Humean mosaic on which everything else is meant to supervene, (ii) the Humean denial of necessary connections, as well as the principle of recombination that goes with it, applies to intrinsically individuated entities, (iii) temporary intrinsics play an important role in Lewis's critique of endurantism.
} 


\section{Being F intrinsically and extrinsically}

The duplication account is inadequate since it specifies that $\mathrm{Fx}_{\mathrm{INT}}={ }_{\mathrm{df}} \mathrm{Fx} \wedge \forall \mathrm{y}(\mathrm{Dyx}$ $\rightarrow$ Fy), while $\mathrm{Fx}_{\mathrm{ExT}}={ }_{\mathrm{df}} \mathrm{Fx} \wedge \exists \mathrm{y}(\mathrm{Dyx} \wedge \neg \mathrm{Fy})$, thereby making $\mathrm{Fx}_{\mathrm{INT}}$ incompatible with $\mathrm{Fx}_{\mathrm{ExT}}$. This is problematic since even though the higher-order properties of being an intrinsic property and of being an extrinsic property are incompatible, the higher-order properties of being had intrinsically by $\mathrm{x}$ and of being had extrinsically by $\mathrm{x}$ are not incompatible. That is, a property $\mathrm{F}$ is either an intrinsic property or an extrinsic property - it cannot be both. Yet, it is not the case that F is either had intrinsically or had extrinsically by $\mathrm{x}-$ it can be both. F can be had both intrinsically and extrinsically by $\mathrm{x}$. The duplication account thus yields an analysis that makes being $\mathrm{F}$ intrinsically and being $\mathrm{F}$ extrinsically incompatible. It thereby mistakenly rules out cases in which a property can be possessed both intrinsically and extrinsically.

An important example of this is the property of being valuable. This is a property that can be had in both of these ways at the same time. A thing can be intrinsically as well as extrinsically valuable. Put differently, having intrinsic value is perfectly compatible with having extrinsic value. A thing can have multiple sources of value, whereby some of these sources can be intrinsic while others can be extrinsic, thereby bringing it about that a thing can have both intrinsic and extrinsic value at the same time. This is something that the duplication account cannot accommodate since it makes being $F$ intrinsically and being $F$ extrinsically incompatible. ${ }^{7}$

Thus, duplication principles are not able to provide an adequate account of intrinsicality since they cannot deal with mixed cases, i.e. cases in which $\mathrm{x}$ is $\mathrm{F}$ both intrinsically and extrinsically. Mixed cases can arise if $\mathrm{F}$ is a disjunctive property involving intrinsic and extrinsic properties, such that Fness can be had both intrinsically and extrinsically if the intrinsic as well as the extrinsic disjuncts are satisfied. If both intrinsic and extrinsic disjuncts can be satisfied, then being $\mathrm{F}$ intrinsically and being $\mathrm{F}$ extrinsically will be compatible. If $\mathrm{x}$ satisfies the intrinsic disjunct, then it is $\mathrm{F}$ wholly in virtue of how it itself is. If in addition $\mathrm{x}$ also satisfies the extrinsic disjunct, then, even though being $\mathrm{F}$ wholly in virtue of how it itself is, $\mathrm{x}$ is not solely $\mathrm{F}$ in virtue of how it itself is since it is also $\mathrm{F}$ in virtue of its extrinsic characteristics. This then makes it the case that $\mathrm{x}$ is both intrinsically $\mathrm{F}$ and extrinsically $\mathrm{F}$. In other words, once the possession of a property can be over-grounded, as happens when a disjunctive property is had in virtue of the first disjunct as well as in virtue of the second disjunct, one needs to distinguish being solely $\mathrm{F}$ in virtue of how a thing itself is from being wholly but not solely

\footnotetext{
${ }^{7}$ Some deny that things are ever extrinsically valuable and instead claim that they are only extrinsically connected to things that are intrinsically valuable (cf. Regan: 2003, pp. 666-667). While this is an interesting position, one should not be committed to this view because of one's views about intrinsicality. Put differently, whether there really is anything such as extrinsic value is a substantive question in value theory that should not be settled by one's account of intrinsicality.
} 
$\mathrm{F}$ in virtue of how a thing itself is. Mixed cases arise when the property is had wholly but not solely in virtue of how the thing itself is. While being F wholly in this way ensures that Fness is had intrinsically, not being had solely in this way ensures that Fness is had extrinsically.

In response, it might be denied that there is one property $\mathrm{F}$ that is had both intrinsically and extrinsically and that there are rather two distinct properties, one of which is had intrinsically while the other is had extrinsically. ${ }^{8}$ In the case at hand, this would amount to denying that there is one property, namely the property of being valuable, that can be had intrinsically as well as extrinsically and that there are instead two distinct properties, namely the property of being intrinsically valuable and the property of being extrinsically valuable. On this proposal, something can have the property of being intrinsically valuable as well as the property of being extrinsically valuable, but it cannot have the property of being valuable both intrinsically and extrinsically. Such an object then has two different properties rather than having one property in two different ways.

While this proposal might be plausible in some cases, it cannot be accepted across the board. In particular, mixed cases will arise when we are dealing with properties the possession of which is the result of a relation that is such that $y$ can be R-related to $\mathrm{x}$ while $\mathrm{x}$ is also R-related to itself. In such cases, $\mathrm{x}$ is $\mathrm{F}$ in virtue of being R-related to itself as well as in virtue of y being R-related to it. One and the same relation gives rise to both the intrinsic possession as well as the extrinsic possession of a property. Put differently, if Fness is both self-conferred and otherconferred, then it is had intrinsically as well as extrinsically. Self-conferral ensures its intrinsic possession, while other-conferral ensures its extrinsic possession.

This can be illustrated by means of a theory of value that considers the possession of the property of being valuable to be the result of a value-conferring process. According to such a theory, $\mathrm{x}$ has the property of being valuable as a result of having value conferred upon it. Mixed cases can then arise if it is possible for something to confer value upon itself as well as to have value conferred upon it by something else. If $\mathrm{y}$ confers value on $\mathrm{x}$ while $\mathrm{x}$ also confers value upon itself, then $\mathrm{x}$ ends up having both intrinsic as well as extrinsic value. In this case, it is not plausible to deny that there is a single property that is had intrinsically as well as extrinsically. This is because the property of being valuable is specified in terms of

\footnotetext{
${ }^{8}$ It should be noted that the possibility of mixed cases has to be admitted if disjunctive properties are accepted since one can then have $\mathrm{F}=_{\mathrm{df}}(\mathrm{I} \vee \mathrm{PE})$, where $\mathrm{I}$ is an intrinsic and PE a pure extrinsic property, making it possible for an object to instantiate this disjunctive property in virtue of instantiating each of the disjuncts, i.e. in virtue of being I as well as in virtue of being PE. Since attempts at making sense of intrinsicality occur in the context of abundant conceptions of properties, the appeal to disjunctive properties should be uncontroversial. The considerations provided in the next paragraphs establish that mixed cases arise not only when dealing with certain gerrymandered disjunctive properties but also with ordinary properties, such as the property of being valuable, being possibly $\mathrm{F}$, having a duplicate etc.
} 
the value-conferring relation that holds between $\mathrm{y}$ and $\mathrm{x}$ as well as between $\mathrm{x}$ and itself. Unlike in cases in which 'intrinsically valuable' and 'extrinsically valuable' are the basic notions and the generic notion of being valuable is derivative, such an account of value ensures that the property of being valuable is prior, whilst the derivative notions are analysed in terms of the core notion of a value-conferring process and then differentiated in terms of whether the value is self-conferred or conferred upon the object by something else.

Similarly, given a counterpart-theoretic account of modality, it is possible for an object to have the same modal property both in virtue of standing in a counterpart relation to itself and in virtue of standing in a counterpart relation to distinct objects. For instance, if $\mathrm{x}$ is $\mathrm{F}$, then $\mathrm{x}$ has the modal property of being possibly $\mathrm{F}$ in virtue of standing in a counterpart relation to itself. In particular, if $\mathrm{x}$ is $\mathrm{F}$ intrinsically, then $\mathrm{x}$ has this modal property intrinsically. Now, if $\mathrm{x}$ also stands in a counterpart relation to an object in another possible world that is also $\mathrm{F}$, then $\mathrm{x}$ also has the property of being possibly $\mathrm{F}$ in virtue of standing in this counterpart relation to this object, which means that $\mathrm{x}$ has this modal property extrinsically. ${ }^{9}$ It consequently has this modal property both intrinsically as well as extrinsically.

A further example of a property giving rise to mixed cases is the property of having a duplicate. This property can be characterised as a property that results from objects standing in the 'being a duplicate of' relation. Since the duplication relation is reflexive, it follows that every thing is such as to ensure that it has a duplicate wholly in virtue of how it itself is. That is, since every thing is its own duplicate, every thing has the property of having a duplicate wholly in virtue of how it itself is. If in addition to having itself as a duplicate, $\mathrm{x}$ also has duplicates that are distinct from it, then it has the property of having a duplicate intrinsically in virtue of being its own duplicate as well as extrinsically in virtue of standing in the 'being a duplicate of' relation to objects mereologically distinct from it. ${ }^{\text {IO }}$

\footnotetext{
${ }^{9}$ Whether and in what way counterpart relations give rise to extrinsicness is a complicated matter and non-standard construals of the counterpart relation might need to be presupposed for this example to work (cf. section 3, especially footnote 20). Instead of bringing in non-standard counterpart relations, one can appeal to the same strategy that is employed in footnote Io and appeal to the property of 'being possibly $\mathrm{F}$ in virtue of facts about a world-mate', which will be had both intrinsically and extrinsically when an object is intrinsically $\mathrm{F}$ and, in addition to being its own counterpart, also has a distinct counterpart in the same world that is F.

ro Since the duplication relation is an internal relation, having a duplicate just amounts to (i) having a certain intrinsic profile and (ii) there being possibilia with the same intrinsic profile. Accordingly, it might be objected that the extrinsicness of this property derives entirely from (ii), yet such quantificational properties regarding the existence of certain possibilia are necessary properties that are usually excluded by combinatorial and duplication analyses. This problem can be circumvented by appealing to the property of having a duplicate that is a world-mate, given that every thing is its own duplicate as well as its own world-mate, which makes it the case that the property is always had intrinsically, and given that the world-mate relation between distinct duplicates is an external relation, which ensures that when the property is also had extrinsically (as a result of an object having a distinct duplicate in the same world) the extrinsicness does not derive from necessary quantificational properties.
} 
Mixed cases thus undermine the duplication analysis. In particular, they require us to reject the left-to-right direction of the $\mathrm{Fx}_{\mathrm{EXT}}$ biconditional. ${ }^{\mathrm{II}}$ Being $\mathrm{F}$ whilst having a duplicate that fails to be $\mathrm{F}$ is a sufficient condition for being $\mathrm{F}$ extrinsically. Yet, it is not a necessary condition given that it is possible for all duplicates to be $\mathrm{F}$ due to the fact that $\mathrm{x}$ is $\mathrm{F}$ both intrinsically and extrinsically.

If we reject absorption principles for properties, i.e. reject that $(F \vee(F \wedge G))$ $=F$ and that $(F \wedge(F \vee G))=F$, then the global versions of the duplication analysis have to be given up as well. Rejecting these absorption principles follows naturally once one grants that an object can be $\mathrm{F}$ both intrinsically and extrinsically.

The property of being both intrinsically and extrinsically valuable is an extrinsic property since it is had partly in virtue of an object's extrinsic features. If we disjoin this property with the property of being intrinsically valuable (or with some other intrinsic property that is implied by the property of being intrinsically valuable), then we end up with a property that can be had extrinsically if it is had in virtue of being both intrinsically and extrinsically valuable. In this way we can specify a property that always holds of objects that have intrinsic value, i.e. any object that has this property is such that all its duplicates are valuable, and sometimes holds of objects that also have extrinsic value, thereby making it the case that the property is had extrinsically in some cases and consequently is extrinsic. Thus, the resulting disjunctive property is extrinsic, since any property that can be had extrinsically is an extrinsic property ${ }^{\mathrm{I} 2}$, yet it never differs amongst duplicates.

Alternatively, if the property of being valuable is had extrinsically in mixed cases, then the property that results from conjoining the property of being valuable with an intrinsic property that is compatible with these mixed cases, such as the property of being intrinsically valuable, can also be had extrinsically. Put differently, if in mixed cases the property of being valuable is had wholly but not solely in virtue of how a thing itself is, then in these cases the property of being valuable and intrinsically valuable is similarly had wholly but not solely in virtue of how a thing itself is.

Accordingly, we can see that such properties undermine the global versions of the duplication analysis. Both the claim that intrinsic properties are properties that never differ amongst duplicates as well as the claim that extrinsic properties are properties that do differ amongst duplicates have to be rejected. Never dif-

\footnotetext{
${ }^{\text {II }}$ It should be noted that the $\mathrm{Fx}_{\mathrm{INT}}$ biconditional is not undermined by mixed cases. This biconditional is only affected by the arguments in the next two sections, which show that we have to reject its right-to-left direction.

${ }^{12}$ This connection between the local and the global notion can be established by the following chain of reasoning: If a property is had extrinsically by $\mathrm{x}$, then it is had at least partly in virtue of how $\mathrm{x}$ is related to other things. If it is had at least partly in virtue of how $\mathrm{x}$ is related to other things, then it is not had solely in virtue of how $\mathrm{x}$ itself is. If it is not a property that is had solely in virtue of how a thing itself is, then it is not an intrinsic property but instead an extrinsic property.
} 
fering amongst duplicates is only a necessary but not a sufficient condition for a property to be intrinsic, whilst differing amongst duplicates is only a sufficient condition but not a necessary condition for a property to be extrinsic.

Combinatorial approaches largely ignore the local notion of intrinsicality and instead focus on the global notion by trying to capture the modal patterns of independence that intrinsic properties exhibit. One possible way of accounting for the local notion combines the combinatorial approach with duplication principles. Langton and Lewis use independence of accompaniment and non-disjunctiveness to identify basic intrinsic properties. These are then used to break the tight definitional circle, allowing them to specify what duplicates are without invoking the notion of intrinsic properties. ${ }^{\mathrm{I}} 3$ That is, independence of accompaniment and non-disjunctiveness yield the basic intrinsic properties, basic intrinsic properties yield duplicates, and duplicates yield intrinsic properties simpliciter. ${ }^{14}$ Once duplicates are specified in terms of basic intrinsic properties, one can use duplication principles to analyse the local notion of intrinsicality. This strategy, however, does not succeed since the duplication approach cannot deal with mixed cases.

We can develop a more promising approach that does not bring in duplication principles, but instead appeals to the notion of a necessitating base. If Fness has both a necessitating base consisting of properties that are members of the set of basic intrinsics (BI) and a necessitating base consisting of properties belonging to the set of basic extrinsics (BE), and if these bases are compatible, then Fness can be had both intrinsically and extrinsically by objects instantiating both of these bases. In this way mixed cases can be accounted for in terms of a property having multiple necessitating bases.

To begin with, we can specify $\mathrm{Fx}_{\mathrm{INT}}$ in terms of $\mathrm{x}$ instantiating certain basic intrinsic properties that necessitate Fness.

$$
\mathrm{Fx}_{\mathrm{INT}}={ }_{\mathrm{df}} \mathrm{Fx} \wedge \exists \mathrm{G}_{\mathrm{G} \in\{\mathrm{BI}\}}(\mathrm{Gx} \wedge \forall \mathrm{y}(\mathrm{Gy} \rightarrow \mathrm{Fy}))^{\mathrm{Is}}
$$

Characterising the necessitating bases of properties had extrinsically is somewhat more difficult. This is because we cannot define being had extrinsically negatively in terms of not having an intrinsic necessitating base since this would rule out

\footnotetext{
${ }^{13}$ The advantage of breaking the definitional circle in this way is that it has fewer theoretical commitments since it only requires judgements of comparative naturalness rather than a commitment to perfectly natural properties (cf. Langton and Lewis: I998).

${ }^{\mathrm{I}}$ Some combinatorial accounts skip the duplication step and specify intrinsic properties as those that supervene on or are truth-functionally compounded out of basic intrinsics (cf. Zimmerman: I997 \& Lewis: 2001). Holistic combinatorial approaches attempt to directly identify the set of intrinsic properties, without a prior identification of the set of basic intrinsic properties (cf. Weatherson: 200 I \& Denby: 2006).

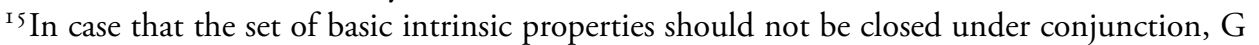
will be a conjunction of properties belonging to the set of basic intrinsic properties rather than $G$ itself being a member of the relevant set. (The same applies, mutatis mutandis, for the subsequent formulations appealing to basic extrinsic properties.)
} 
mixed cases. Instead, we have to provide a positive specification. This can be done straightforwardly when dealing with properties that have a pure extrinsic base, i.e. x's being had purely extrinsically can be specified in terms of $x$ instantiating certain basic extrinsic properties that necessitate Fness. ${ }^{16}$

$$
\mathrm{Fx}_{\mathrm{PURE}-\mathrm{EXT}}=\mathrm{df}_{\mathrm{df}} \mathrm{Fx} \wedge \exists \mathrm{G}_{\mathrm{G} \in\{\mathrm{BE}\}}(\mathrm{Gx} \wedge \forall \mathrm{y}(\mathrm{Gy} \rightarrow \mathrm{Fy}))
$$

With this in place, we can account for impure disjunctive properties of the form $\mathrm{F}=\mathrm{df}_{\mathrm{df}}(\mathrm{I} \vee \mathrm{PE})$. Such properties have both an intrinsic and a pure extrinsic base, which implies that an object has such a property both intrinsically and extrinsically if it has both bases. This, however, still leaves the other impure cases. Impure conjunctive cases of the form $\mathrm{F}==_{\mathrm{df}}(\mathrm{I} \wedge \mathrm{PE})$ can be defined in terms of Fness having neither an intrinsic nor a pure extrinsic necessitating base.

$$
\begin{aligned}
& \mathrm{Fx}_{\wedge \text {-ExT }}=\mathrm{df}_{\mathrm{df}} \wedge \neg \exists \mathrm{G}_{\mathrm{G} \in\{\mathrm{BI}\}}(\mathrm{Gx} \wedge \forall \mathrm{y}(\mathrm{Gy} \rightarrow \mathrm{Fy})) \wedge \neg \exists \mathrm{H}_{\mathrm{H} \in\{\mathrm{BE}\}}(\mathrm{Hx} \\
& \wedge \forall \mathrm{z}(\mathrm{Hz} \rightarrow \mathrm{Fz}))
\end{aligned}
$$

Disjunctive impure properties that have the form $F={ }_{d f}\left(I_{I} \vee\left(I_{2} \wedge P E\right)\right)$ and which also admit of mixed cases, however, give rise to yet further difficulties. Having such a property extrinsically needs to be specified in terms of having the impure conjunctive necessitating base of a property that also has a purely intrinsic necessitating base.

$$
\begin{aligned}
& \mathrm{Fx}_{\mathrm{V} \text {-EXT }}=\mathrm{df}_{\mathrm{df}} \wedge \exists \mathrm{G}_{\mathrm{G} \in\{\mathrm{BE}\}} \exists \mathrm{H}_{\mathrm{H} \in\{\mathrm{BI}\}}(\mathrm{Gx} \wedge \mathrm{Hx} \wedge \forall \mathrm{y}(\mathrm{Gy} \wedge \mathrm{Hy} \rightarrow \mathrm{Fy}) \\
& \wedge \neg \forall \mathrm{z}(\mathrm{Gz} \rightarrow \mathrm{Fz}) \wedge \neg \forall \mathrm{v}(\mathrm{Hv} \rightarrow \mathrm{Fv})) \wedge \exists \mathrm{I}_{\mathrm{I} \in\{\mathrm{BI}\}} \forall \mathrm{w}(\mathrm{Iw} \rightarrow \mathrm{Fw})
\end{aligned}
$$

Though this way of characterising the local notion of intrinsicality is superior to an account that appeals to duplication principles, this alternative approach faces two problems. The first problem is that in order for this strategy to work we need to know which properties belong to the sets of basic intrinsic and basic extrinsic properties. Yet, this cannot be achieved solely by means of logical, modal, mereological and spatio-temporal notions. As a result, we need some additional resources that allow us to provide an ordering of properties.

The second problem is that the necessitation relation is not sufficiently finegrained to deal with extensionally equivalent properties. On the one hand, this is problematic in that it leads to the misguided commitment that every disjunctive property admits of mixed cases. This problem arises because the proposed account cannot distinguish (I $\vee \mathrm{PE})$ from $(\mathrm{I} \vee(\neg \mathrm{I} \wedge \mathrm{PE})$ ). While the former property allows for mixed cases, the latter property is either had intrinsically (in virtue of I)

\footnotetext{
${ }^{16}$ If we partition all possibilia into equivalence classes under the relation of duplication, then those properties that neither include nor exclude any equivalence classes but divide all classes are pure extrinsic properties. In addition to the pure extrinsic properties, there are three kinds of impure cases, namely (i) those that include some, divide some, exclude none, (ii) those that include none, divide some, exclude some, and (iii) those that include some, divide some, exclude some (cf. Lewis: I983a, p. 356 footnote I6). A hyperintensional characterisation of these different types of extrinsic properties is given below (cf. section 5.3).
} 
or extrinsically (in virtue of $\neg \mathrm{I}$ and PE) yet is never had both intrinsically and extrinsically. Relatedly, it is unable to deal with all those mixed cases that involve the failure of absorption principles since this account cannot distinguish $\left(I_{I} \vee\left(I_{2}\right.\right.$ $\wedge P E)$ ) from $I_{I}$ where the extension of $I_{2}$ is a subset of the extension of $I_{I}$. On the other hand, this gives rise to the problem that if we were to adopt this strategy, then we would end up with a situation in which necessary properties would always be had both intrinsically and extrinsically since every property whatsoever necessitates all necessary properties, thereby ensuring that they would have both intrinsic and extrinsic necessitating bases. Since properties that can be had extrinsically are extrinsic properties, it would follow from this account that all necessary properties are extrinsic. This outcome is unacceptable to defenders of combinatorial approaches since combinatorial principles imply that all necessary properties are intrinsic. Put differently, appealing to necessitating bases in order to specify the local notion of intrinsicality leads to direct conflicts with the combinatorial accounts of the global notion of intrinsicality. Accordingly, we can see that we need something more fine-grained than the necessitation relation, namely a grounding relation that tracks hyperintensional connections (cf. section 5.2).

Thus, neither the duplication account nor the combinatorial account developed in terms of the notion of a necessitation base is successful when it comes to the local notion of intrinsicality since neither of these accounts is able to deal with mixed cases. This is problematic for two reasons.

First, the local notion performs important philosophical work, for instance it is this notion that is at issue when one is concerned with intrinsic/extrinsic value, and accordingly is something that needs to be accounted for. This means that there is a need to find an alternative theory that can deal with mixed cases, in particular a hyperintensional theory of the local notion that allows us to distinguish different ways in which a property can be had, thereby making room for the distinction between being F solely in virtue of how a thing itself is and being F wholly but not solely in virtue of how a thing itself is.

Second, as we noted above, once mixed cases are accepted it is natural to reject absorption principles and instead hold that necessarily co-extensive properties can differ in whether they are intrinsic. This ensures that the inability to deal with the local notion also undermines the account of the global notion. For instance, the disjunctive property $(F \vee(F \wedge G))$, where $F$ is intrinsic and $G$ is extrinsic, is extensionally equivalent to $\mathrm{F}$, yet the former can be had both intrinsically as well as extrinsically whilst the latter is always only had intrinsically. Non-hyperintensional approaches are unable to deal with such disjunctive properties and should consequently be rejected. 


\section{Extra-world sources of extrinsicness}

A further problem for duplication and combinatorial accounts is that analysing intrinsicality in extensional terms does not allow us to adequately assess the intrinsicality of modal properties. This is because an extensional analysis ignores extra-world sources of extrinsicness. ${ }^{17}$ This leads to problems in that extensional accounts classify modal properties as being intrinsic as long as they are not determined by extrinsic determinants that are to be found in the same world. Since these accounts only track intra-world dependence, problematic consequences arise when it comes to modal properties that are intra-world independent but nonetheless cross-world dependent. These properties are extrinsic but are nonetheless classified as being intrinsic by duplication and combinatorial accounts.

In particular, these accounts misclassify certain modal properties when these are construed along counterpart-theoretic lines. According to counterpart theory, $\mathrm{x}$ is possibly $\mathrm{F}$ iff $\mathrm{x}$ has a counterpart that is $\mathrm{F}$. This means that an object's modal properties derive from its standing in counterpart relations to distinct entities in distinct possible worlds. ${ }^{18}$ This makes the possession of such modal properties an extrinsic matter. Yet, certain of these modal properties do satisfy the duplication principles as well as the combinatorial conditions. These modal properties do not differ amongst duplicates. They are independent of accompaniment (and non-disjunctive). They supervene on intrinsic non-modal properties. Yet they are extrinsic.

It should be noted that counterpart theory makes the possession of many modal properties dependent on extrinsic factors to be found in the same world. This is because the similarity relations which determine counterpart relations take into account the sortals of the objects and these sortals are often extrinsically sensitive. For example, duplicates can differ in what counterparts they have if they differ in whether a sortal $\mathrm{F}$ applies to them, given that Fness has extrinsic determiners. This happens for example if Fness is subject to maximality conditions, which makes it possible that $\mathrm{x}$ is $\mathrm{F}$ even though its duplicate $\mathrm{y}$ fails to be $\mathrm{F}$ since only $\mathrm{x}$ is a maximal F-candidate while $\mathrm{y}$ is a proper part of a thing that is an $\mathrm{F}^{19}$

\footnotetext{
${ }^{17}$ This is particularly clear when it comes to combinatorial accounts since they are only concerned with intra-world compossibility, thereby ignoring extra-world sources of extrinsicality. The notions of loneliness and accompaniment are intra-world rather than cross-world notions. By assessing whether a property is independent of accompaniment one only assesses the relations it bears to its worldmates, but leaves aside the relations it bears to objects in other possible worlds.

${ }^{18}$ Counterpart theory allows for exceptions to this claim which arise from modal properties that objects have as a result of standing in counterpart relations to actual-world counterparts. If $\mathrm{x}$ is $\mathrm{F}$, then $\mathrm{x}$ has the modal property 'being possibly $\mathrm{F}$ ' in virtue of having an actual-world counterpart (namely itself) that is F. However, this does not work for the property 'being $\neg \mathrm{F}$ but possibly F' (excepting cases of multiple actual-world counterparts) or for the property 'being necessarily F'.

${ }^{19}$ The combinatorial account proposed by Langton and Lewis has difficulties in dealing with maximal properties (cf. Sider: 200I), but more sophisticated combinatorial analyses, such as the one proposed by Weatherson, do not suffer from this defect (cf. Weatherson: 200I, p. 372).
} 
Duplication principles can adequately deal with these modal properties since the source of extrinsicness is to be found in the same world. Yet, certain modal properties are misclassified as being intrinsic, namely those where maximality and other extrinsic conditions do not apply. In other words, in cases where the counterpart relation is not affected by extrinsic factors, but is only determined by the intrinsic properties of the relata, the modal property should be classified as being extrinsic given that it depends on the relation the thing has to a distinct object, but is mistakenly classified as being intrinsic. ${ }^{20}$

This problem is brought out by the differential verdicts that are given when assessing the intrinsicality of temporal and modal properties, when both of these kinds of properties are given a counterpart-theoretic analysis. Stage theorists claim that $\mathrm{x}$ has the temporal property wAS/wILL be $\mathrm{F}$ iff $\mathrm{x}$ has a past/future temporal counterpart that is $\mathrm{F}$. This implies that an object's temporal properties are extrinsic properties since they are dependent on the properties of its temporal counterparts. Duplicates of stages can differ in terms of their historical and futural properties because they can differ in what temporal counterparts they have, implying that these properties are extrinsic. That is, a lonely stage lacks the temporal property WAS $\mathrm{F}$ even though an intrinsic duplicate can have that property if it is accompanied by a past temporal counterpart that is $\mathrm{F}^{21}$

Modal and temporal counterpart theory agree in that modal/temporal properties are had by an object in virtue of that object standing in the relevant relations to distinct entities. The difference between them is whether the counterparts are to be found in different worlds or in the same world but at different times. ${ }^{22}$

\footnotetext{
${ }^{20}$ Standard construals of the counterpart relation that treat this relation as being based on facts about similarity make it the case that, in those situations in which the counterpart relation supervenes on the intrinsic properties of the relata, the property of having counterparts boils down to the conjunctive property of being $F$ and such that there exist possibilia that are $G$, where $F$ and $\mathrm{G}$ are the intrinsic properties of the relata of the counterpart relation. Such a property is the conjunction of a contingent intrinsic property (being F) and an extrinsic necessary quantificational property (being such that there exist possibilia that are G). If such a construal of the counterpart relation is accepted, then the extrinsicality of the modal properties resulting from these internal counterpart relations is entirely due to the extrinsic necessary conjunct. Put differently, these internal counterpart relations do not give rise to any extrinsicality beyond the commitment to the existence of the relata. Accordingly, the problem outlined in this section would simply be an instance of the general problem that duplication and combinatorial approaches are unable to give a proper account of necessary properties. What is needed to generate a new problem is a case in which the counterpart relation supervenes on the intrinsic properties of the relata without being grounded in those properties. In other words, what is needed is an account, such as Ramachandran's Kripkean counterpart theory, that treats the counterpart relation as an external relation, as a 'substantial' cross-world relation, that in certain cases can nonetheless be necessarily co-extensive with an internal relation.

${ }^{21}$ Similarly, historical and futural properties are not independent of accompaniment on this account and consequently are not intrinsic properties. A lonely stage cannot have the property 'WAS/WILL be F' since there is no past/future temporal counterpart that is F.

${ }^{22}$ In addition, differences can arise when it comes to the nature of the counterpart relation.
} 
Nonetheless, extensional accounts give different verdicts as to the intrinsicality of some of these properties. Yet, whether the counterparts are in the same world or in other worlds should not affect the intrinsicality status since in each case the properties result from relations to distinct things. We can thus see that while future- and past-directed ${ }^{23}$ temporal properties are correctly judged to be extrinsic, modal properties that supervene on the intrinsic properties of the relata of the counterpart relation are misclassified as being intrinsic.

Thus, counterpart-theoretic modal properties are extrinsic insofar as an object possesses such a modal property in virtue of relations that it bears to other objects. It is not a property that it has solely in virtue of how it itself is (except insofar as the modal property is solely based on a counterpart relation the thing bears to itself). Similarly, temporal properties construed along counterpart-theoretic lines (other than those made true solely by counterpart relations the thing bears to itself) are extrinsic properties since they depend on relations to distinct objects. Duplication and other extensional accounts can adequately deal with pastand future-directed temporal properties ${ }^{24}$ but problems arise in the case of modal properties. In particular, these accounts mistakenly classify those modal properties as intrinsic that are intra-world independent but cross-world dependent. The reason for this problem is that extensional accounts are unable to identify extra-world sources of extrinsicness.

\section{Boolean closure and necessary/impossible properties}

Combinatorial and duplication approaches face problems in dealing with necessary and impossible properties, which has led a number of proponents of these approaches to exclude such properties from their analyses. ${ }^{25}$ In particular, adopting an extensional understanding of property instantiation and individuation has

While standard accounts of the modal counterpart relation construe it as being based on facts about similarity, temporal counterpart relations are frequently understood as being based on more 'substantive' relations, such as relations of immanent causation. As mentioned in footnote 20, non-standard construals of the modal counterpart relation need to be brought in if the argument in this section is not to rely on appealing to the extrinsicality of necessary properties.

${ }^{23}$ We need to exclude temporal properties involving only Now-operators since these properties are self-conferred, given that present-counterparts function as truth-makers, and are consequently classified as intrinsic by extensional accounts.

${ }^{24}$ Problems regarding mixed properties arise in the temporal case as well. This is because the property of being $\mathrm{F}$ at some time can be had both intrinsically by $\mathrm{x}$ (if $\mathrm{x}$ is intrinsically $\mathrm{F}$ ) and extrinsically by $\mathrm{x}$ (if $\mathrm{x}$ also has past or future counterparts that are $\mathrm{F}$ ).

${ }^{25}$ In a similar manner, non-qualitative properties have also frequently been excluded. For criticisms of attempts to avoid counter-examples based on identity properties and necessary properties by restricting the scope of combinatorial and duplication accounts cf. Eddon: 20I I. Eddon concludes from the failure of the restriction strategy that an account of intrinsicality needs to be sensitive to hyperintensional distinctions, but then ends up taking 'intrinsic' to be primitive, rather than providing a hyperintensional analysis. 
the consequence that all necessary properties and all impossible properties have the same status (since there really is only one necessary property and one impossible property on this view). This is problematic since we seem to be committed to there being both intrinsic and extrinsic necessary properties. In order to make sense of such a commitment we need to bring in hyperintensional resources. Given that these properties are extensionally equivalent, they can differ only hyperintensionally.

That there are both intrinsic and extrinsic necessary properties follows from certain Boolean closure principles, in particular the principle that the sets of intrinsic and pure extrinsic properties are closed under Boolean combinations. To say that the set of intrinsic properties is closed under Boolean operations is to say that if $F$ and $G$ are intrinsic properties, then all properties formed from $F$ and $G$ by means of Boolean connectives will also be intrinsic, such as the negative properties $\neg \mathrm{F}$ and $\neg \mathrm{G}$, as well as the conjunctive property $(\mathrm{F} \wedge \neg \mathrm{G}$ ) and the disjunctive property $(F \vee G)$. It should be noted that, amongst the extrinsic properties, only the set of pure extrinsic properties satisfies the closure conditions and that the set of impure extrinsic properties is not closed in this way.

The problem now is that it follows from these closure principles that extensionally equivalent properties differ in intrinsicality status. In particular, it follows from I's being intrinsic that the property $\neg \mathrm{I}$ as well as the disjunctive property (I $\vee \neg I$ ) will also be intrinsic. Similarly, it follows from PE's being a pure extrinsic property that the property $\neg \mathrm{PE}$ as well as the disjunctive property (PE $\vee \neg \mathrm{PE}$ ) will also be pure extrinsic properties. For example, the property of being lonely is a pure extrinsic property. By Boolean closure, being lonely or not lonely is a pure extrinsic property. Yet being lonely or not lonely is a necessary property. Accordingly, we end up with two necessary properties, namely (I $\neg \neg \mathrm{I})$ and (PE $\vee \neg \mathrm{PE}$ ), that are extensionally equivalent, whereby one is intrinsic and the other extrinsic.

Proponents of extensional accounts consequently face a dilemma. They either have to give up the closure conditions or give up extensionality and instead allow for hyperintensionality. Giving up closure principles is problematic. In particular, the problem is that it simply does not seem possible to end up with an intrinsic property by combining different pure extrinsic properties. How can we construct an intrinsic property if all that is at our disposal are pure extrinsic properties? In what way can we make the extrinsicness disappear? Similarly, it seems that we cannot end up with an extrinsic property by combining different pure intrinsic properties. Yet, impossible properties result from combining $\mathrm{F}$ and $\neg \mathrm{F}$ both if $\mathrm{F}$ and $\neg \mathrm{F}$ are intrinsic and if they are extrinsic. Similarly, necessary properties result from disjoining $\mathrm{F}$ and $\neg \mathrm{F}$ both if $\mathrm{F}$ and $\neg \mathrm{F}$ are intrinsic and if they are extrinsic.

Additionally, there are independent motivations for rejecting extensionality 
insofar as there seem to be both intrinsic and extrinsic necessary properties. When we are dealing with disjunctive properties, it seems plausible to say that these can be had in different ways, namely in virtue of the first disjunct and/or in virtue of the second disjunct. ${ }^{26}$ Accordingly, there is a difference between disjunctive necessary properties that have intrinsic disjuncts and those that have extrinsic disjuncts. The former are had in virtue of the intrinsic properties of a thing, while the latter are had in virtue of the extrinsic properties of a thing. These disjunctive necessary properties can be had in different ways, which means that there can be hyperintensional differences amongst extensionally equivalent properties.

The problem with extensional understandings of properties is that we need to know not only whether a disjunctive property is had but also how it is had. Extensional principles break down since we have different disjunctive necessary properties that differ not in whether they are had but in how they are had. Whether a disjunctive necessary property is had in virtue of the first disjunct or in virtue of the second disjunct is a contingent matter. In other words, when dealing with this kind of disjunctive necessary property, it is necessary that this property is had but it is contingent how it is had.

Given that we accept both intrinsic and extrinsic necessary properties, we also end up with contingent properties that are extensionally equivalent but are nonetheless distinct and differ in terms of their intrinsicality status. For example, if we accept that there are extrinsic disjunctive properties that are necessary properties, then the conjunction of I and (PE $\vee \neg \mathrm{PE}$ ) will not be intrinsic even though it is extensionally equivalent to an intrinsic property. This is because the conjunction of an intrinsic property I and an extrinsic property (PE $\vee \neg \mathrm{PE}$ ) does not yield an intrinsic property, but rather an extrinsic property. In other words, the conjunctive property $\mathrm{F}={ }_{\mathrm{df}}(\mathrm{I} \wedge(\mathrm{PE} \vee \neg \mathrm{PE}))$ can be had in different ways, whereby extrinsic features can affect the way in which Fness is had by $\mathrm{x}$. Though it is the case that whether $\mathrm{x}$ is $\mathrm{F}$ does not depend on whether $\mathrm{x}$ is PE, since $\mathrm{x}$ would have been $\mathrm{F}$ even if it had not been PE, its being PE does have an effect on how $\mathrm{x}$ is $\mathrm{F}$. Its being $\mathrm{F}$ in a particular way depends on its being PE. Whether $\mathrm{x}$ is $\mathrm{F}$ because of its being I and PE, or because of its being I and $\neg \mathrm{PE}$, is determined not only by the intrinsic properties of $\mathrm{x}$, but also by its extrinsic properties.

The closure principles also seem to imply that there are both intrinsic and extrinsic impossible properties, i.e. $(\mathrm{I} \wedge \neg \mathrm{I})$ as well as $(\mathrm{PE} \wedge \neg \mathrm{PE})$. Yet, we cannot appeal to hyperintensional differences amongst these properties since they are not disjunctive properties that can be had in different ways. Such conjunctive properties cannot be had in different ways since they are had in virtue of both

\footnotetext{
${ }^{26}$ This only holds for genuinely disjunctive properties. For example, the property $(\mathrm{F} \vee \mathrm{F})$ collapses to the property $\mathrm{F}$. This is because this 'disjunctive' property can only be had in one way. This means that $\mathrm{F}$ is not only extensionally equivalent to $(\mathrm{F} \vee \mathrm{F})$ but also equivalent according to our hyperintensional criterion (cf. section 5.I).
} 
conjuncts if they are had at all.

Instead of appealing to hyperintensional differences, we have to argue that impossible properties are not had at all and are consequently neither intrinsic nor extrinsic. This means that we cannot accept unrestricted closure principles but have to impose the restriction that the property must be had at all if it is to inherit its intrinsicality status from its constituents (cf. section 5.2). In particular, while we can accept upward-closure, we have to reject downward-closure since the lower bound neither belongs to the set of intrinsic nor the set of pure extrinsic properties. This, however, is unproblematic since the rejection of downwardclosure is well motivated and does not conflict with the reasons we gave in support of accepting closure. The motivation for accepting closure is that it does not seem plausible to say that we can combine intrinsic properties to yield an extrinsic property and that we can combine pure extrinsic properties to yield an intrinsic property. This is neither undermined by arguing that pure extrinsic properties can be combined to yield an impossible property that is neither intrinsic nor extrinsic, nor by arguing that the same holds, mutatis mutandis, for intrinsic properties.

Moreover, this restriction can be justified since, according to the 'in virtue of' theory, the fundamental question to ask is how properties are had. That is, the key question is not whether properties are intrinsic or extrinsic properties, but whether they are had intrinsically or extrinsically. Whether a property is intrinsic or extrinsic is a derivative question that is determined by whether it is always had only intrinsically or at least sometimes extrinsically. Accordingly, we can ignore impossible properties since they are never had at all. Since they are not had at all, they are had neither intrinsically nor extrinsically and hence are neither intrinsic nor extrinsic properties. ${ }^{27}$

The resulting understanding of closure is symmetrical with respect to intrinsic and pure extrinsic properties. In both cases we accept closure principles as long as they do not yield an impossible property. Lewis et al., however, need to treat intrinsic and pure extrinsic properties in an asymmetrical manner. They accept full closure for the set of intrinsic properties, while restricting upward- as well as downward-closure for the set of pure extrinsic properties since both the upper as well as the lower bound are classified as intrinsic rather than pure extrinsic.

${ }^{27}$ When giving duplication accounts, we can also impose a restriction by specifying that these principles only apply to non-empty properties (cf. Humberstone: I 996). However, it is not clear whether the duplication framework has the relevant resources to provide a justification for this restriction. It is only by taking the question of how properties are had as basic that we are able to explain why we are dealing only with non-empty properties when distinguishing intrinsic from extrinsic properties since only non-empty ones are had at all. 


\section{Towards an 'in virtue of' theory}

Having established the inadequacy of combinatorial and duplication approaches, we can now identify some of the features that an adequate theory of intrinsicality needs to possess. In particular, it must be (i) a hyperintensional theory that appeals to in virtue of claims, (ii) a theory that distinguishes different ways in which a disjunctive property can be had and provides refined versions of supervenience, duplication and closure principles that take this into account, and (iii) a theory that takes the local notion of intrinsicality as more basic than the global notion.

\section{I Hyperintensionality: 'in virtue of'}

We started with two platitudes, namely (i) that intrinsic properties are possessed solely in virtue of the way things themselves are, and (ii) that the instantiation of intrinsic properties is independent of how the rest of the world is (intrinsically). Combinatorial and duplication accounts have focused on the second platitude by trying to capture the patterns of modal independence exhibited by intrinsic properties or by assessing the distribution of properties across duplicates. Not only have they focused on the second platitude at the expense of the first, they have also misinterpreted the content of this platitude. By construing the notion of instantiation in a narrow sense that considers only whether a property is had but not how it is had, they have ignored the fact that the independence platitude should be understood as stating not only that whether intrinsic properties are instantiated is independent of how the rest of the world is (intrinsically) but also that the way such properties are instantiated is independent in this way. By doing so they have ignored an important hyperintensional element of this platitude. Rather than adopting an extensional approach and supplementing it with considerations of naturalness, we should instead give a hyperintensional theory of intrinsicality that appeals to 'in virtue of' claims. Such an account allows us to take the platitudes with which we started at face value.

In the previous sections we saw that appealing to naturalness does not enable us to provide a satisfactory account of intrinsicality and that hyperintensional resources must be brought in. This is because naturalness does not affect our understanding of how properties are instantiated and individuated. Naturalness only tells us what kind of property we are dealing with, in particular what degree of naturalness it has, but it does not affect the manner in which properties are instantiated. Put differently, naturalness only provides us with an ordering of properties and affects neither property identity criteria nor property instantiations. ${ }^{28}$

\footnotetext{
${ }^{28}$ For instance, on a Lewisian account, properties are considered as sets of possibilia and criteria for property identity and property instantiations are specified extensionally. Instantiation is understood as set-membership, i.e. Fx iff $\mathrm{x} \in\{\mathrm{F}\}$, making it the case that instantiation $\phi(\mathrm{Fx})$ = instantiation $\psi(\mathrm{Gy})$ iff the subject of instantiation is the same, i.e. $\mathrm{x}=\mathrm{y}$, and the property
} 
Rather than appealing to 'naturalness', we have to make use of the 'in virtue of' notion. This notion allows us to distinguish different ways in which a property can be had, thereby making room for a hyperintensional account of property instantiation which then leads to a hyperintensional criterion for property identity.

More precisely, a hyperintensional criterion of property instantiation makes our account more fine-grained, allowing there to be different ways in which one and the same property can be had. An object $x$ can be $F\left(\right.$ where $F={ }_{\text {df }}(P E \vee \neg P E)$ ) in virtue of being $\mathrm{PE}$, while another object y can be $\mathrm{F}$ in virtue of being $\neg \mathrm{PE}$. Accordingly, we should not only assess whether Fness is instantiated, whether Fness is had, but how this property is instantiated, how it is had. Extensional accounts merely record the fact that $\mathrm{x}$ is $\mathrm{F}$ and that $\mathrm{y}$ is $\mathrm{F}$, but they cannot distinguish the ways in which these objects are $\mathrm{F}$.

Property instantiation should not merely be construed as a pair consisting of an object and a property, but should also include the manner in which a property is instantiated. The manner of instantiation is understood in terms of that which grounds the instantiation of the property. We should not only look at whether Fness is instantiated, but also look at that in virtue of which Fness is instantiated. This means that a property can be had in different ways if it can have different grounds. This makes room for property instantiations $\phi$ and $\psi$ of the same property $\mathrm{F}$ by the same object $\mathrm{x}$ at the same time $\mathrm{t}$ to be distinct given that $\mathrm{x}$ instantiates $\mathrm{F}$ at $\mathrm{t}$ in different ways. As a result, it is possible to account for mixed cases, allowing Fness to be both grounded in an intrinsic source as well as in an extrinsic source.

This hyperintensional account of property instantiations in turn gives rise to a hyperintensional account of properties. We end up with a hyperintensional criterion for the identity of properties that individuates properties in terms of how they can be instantiated. Property identity requires not only extensional equivalence but also hyperintensional equivalence. That is, properties must be instantiated in the same ways by the same particulars for them to be identical. Accordingly, properties $\mathrm{F}$ and $\mathrm{G}$ can be distinct even though they are extensionally equivalent. ${ }^{29}$ For properties $\mathrm{F}$ and $\mathrm{G}$ to be identical, they must be had by the same things and must be instantiated in the same ways.

Though this theory of properties is hyperintensional, it is not an ultra fine-

being instantiated is the same, i.e. $F=G$. Property $F=$ property $G$ iff $\forall x(x \in\{F\} \leftrightarrow x \in\{G\})$. Naturalness does not appear in either of these conditions. If one gives up extensionality, then one can develop theories of properties where naturalness plays a more important role and not only specifies an ordering on properties but also affects how properties are instantiated and individuated, e.g. property $\mathrm{F}=$ property $\mathrm{G}$ iff (i) $\forall \mathrm{x}(\mathrm{Fx} \leftrightarrow \mathrm{Gx})$ and (ii) $\mathrm{F}$ and $\mathrm{G}$ are equally natural, i.e. property identity then requires both extensional equivalence and equivalence of naturalness. Yet, as long as hyperintensionality is rejected, naturalness will not allow us to distinguish extensionally equivalent properties (since they will be identical on such a view) and will not allow us to distinguish different ways in which one and the same property can be had.

${ }^{29}$ Witmer, Butchard and Trogdon also note that "properties that are necessarily coextensive can yet differ in whether they are intrinsic" (Witmer, Butchard and Trogdon: 2005, p. 334). 
grained theory. In particular, associativity, commutativity, distributivity and idempotency principles hold. ${ }^{30}$ This means, for instance, that $(F \vee G)$ is the same property as $(\mathrm{G} \vee \mathrm{F})$, and that $(\mathrm{F} \wedge \mathrm{F})$ is the same property as $\mathrm{F}$. This is because the criterion for property identity is insensitive to repetitions and to the order of the constituents of a property.

Lewis notes that "[i]f we had a clear enough understanding of 'solely in virtue of', we would need no further definition of 'intrinsic'." (Lewis: 2001, p. 384) The preceding arguments show that a satisfactory account of intrinsicality must appeal to 'in virtue of' claims. Since the 'in virtue of' locution cannot be analysed but has to be taken as primitive, it follows that it is not possible to give a 'further definition' of 'intrinsic' that does not explicitly rely on this notion. This, however, does not mean that all we can do is to state the platitude that intrinsic properties are possessed solely in virtue of how a thing itself is. Instead, we can provide various principles and constraints that rely on 'in virtue of' claims and which allow us to properly characterise the notion of intrinsicality.

\subsection{Ways of instantiating properties}

Given this hyperintensional understanding of property instantiation and individuation, we should reject the interdefinability of duplication and intrinsicality in both the local and the global case. In the local case, we should reject the biconditional that $\mathrm{x}$ is $\mathrm{F}$ intrinsically iff all duplicates of $\mathrm{x}$ are F. Instead, we should only accept the left-to-right direction, namely that all duplicates being $\mathrm{F}$ is necessary but not sufficient for $\mathrm{x}$ to be $\mathrm{F}$ intrinsically. We should also reject the biconditional that $\mathrm{x}$ is $\mathrm{F}$ extrinsically iff $\mathrm{x}$ is $\mathrm{F}$ and there is some duplicate of $\mathrm{x}$ that fails to be F. Instead, we should only accept the right-to-left direction, namely that there being a duplicate that fails to be $\mathrm{F}$ is sufficient but not necessary for $\mathrm{x}$ being F extrinsically.

$$
\begin{array}{ll}
\text { reject: } & \mathrm{Fx}_{\mathrm{INT}} \leftrightarrow \mathrm{Fx} \wedge \forall \mathrm{y}(\mathrm{Dyx} \rightarrow \mathrm{Fy}) \\
\text { accept: } \mathrm{Fx}_{\mathrm{INT}} \rightarrow \mathrm{Fx} \wedge \forall \mathrm{y}(\mathrm{Dyx} \rightarrow \mathrm{Fy}) \\
\text { reject: } \mathrm{Fx}_{\mathrm{EXT}} \leftrightarrow \mathrm{Fx} \wedge \exists \mathrm{y}(\mathrm{Dyx} \wedge \neg \mathrm{Fy}) \\
\text { accept: } \mathrm{Fx}_{\mathrm{EXT}} \leftarrow \mathrm{Fx} \wedge \exists \mathrm{y}(\mathrm{Dyx} \wedge \neg \mathrm{Fy})
\end{array}
$$

In the global case, we should also reject the biconditionals. As we have seen, there are properties that never differ amongst duplicates but are yet extrinsic since they can be had extrinsically. Accordingly, while it is true that intrinsic properties never differ amongst duplicates, it is not the case that all properties that never differ amongst duplicates are intrinsic. Not differing amongst duplicates is not sufficient for a property to be intrinsic. Similarly, while it is true that properties

\footnotetext{
${ }^{30}$ As we saw above, absorption fails: $(F \wedge(F \vee G))$ is distinct from $F$ since the former property can be had in virtue of being $(F \wedge G)$, whilst the latter can only be had in virtue of being $F$.
} 
that differ amongst duplicates are extrinsic, it is not the case that all extrinsic properties are such that they differ amongst duplicates. Differing amongst duplicates, though sufficient for being an extrinsic property, is not a necessary condition for a property to be extrinsic.

$$
\begin{array}{ll}
\text { reject: } & \mathrm{F} \in\{\mathrm{I}\} \leftrightarrow \forall \mathrm{x}(\mathrm{Fx} \rightarrow \forall \mathrm{y}(\mathrm{Dyx} \rightarrow \mathrm{Fy})) \\
\text { accept: } & \mathrm{F} \in\{\mathrm{I}\} \rightarrow \forall \mathrm{x}(\mathrm{Fx} \rightarrow \forall \mathrm{y}(\mathrm{Dyx} \rightarrow \mathrm{Fy})) \\
\text { reject: } & \mathrm{F} \in\{\mathrm{E}\} \leftrightarrow \exists \mathrm{x}(\mathrm{Fx} \wedge \exists \mathrm{y}(\mathrm{Dyx} \wedge \neg \mathrm{Fy})) \\
\text { accept: } \mathrm{F} \in\{\mathrm{E}\} \leftarrow \exists \mathrm{x}(\mathrm{Fx} \wedge \exists \mathrm{y}(\mathrm{Dyx} \wedge \neg \mathrm{Fy}))
\end{array}
$$

Similarly, we should reject the supervenience principle that properties that supervene on intrinsic properties are themselves intrinsic. For example the property (I $\wedge(\mathrm{PE} \vee \neg \mathrm{PE}))$ is an extrinsic property, given a hyperintensional characterisation of intrinsicality, yet it supervenes on the intrinsic property I. Thus, while it is true that (non-basic) intrinsic properties supervene on (basic) intrinsic properties, it is not the case that all properties that supervene on intrinsic properties are themselves intrinsic. The supervenience of A-properties on intrinsic B-properties is necessary but not sufficient for the A-properties to be intrinsic. The failure of supervenience of A-properties on intrinsic B-properties is sufficient but not necessary for the A-properties to be extrinsic.

While these traditional principles and analyses need to be rejected, we can devise refined versions thereof that incorporate the relevant hyperintensional elements. These more fine-grained principles do not only assess whether a property is had but also take into consideration how it is had.

DUPLICATION: As we saw above, the traditional duplication account is inadequate since it cannot deal with mixed cases as well as with necessary extrinsic properties. The problem was that an extrinsic property can be such as to not differ amongst duplicates. This was either due to the fact that the property was also had intrinsically by a particular x (local mixed case) or by all x (global mixed case), or due to the fact that the property was a necessary property.

This problem can be avoided by construing duplication principles not only as assessing whether duplicates share a particular property but also whether duplicates have this property in the same way. While an extrinsic property can be such as to not differ amongst duplicates, it cannot be such that all duplicates have that property in the same way. Accordingly, we do not just specify whether either all duplicates or only some duplicates of a particular $\mathrm{x}$ that is $\mathrm{F}$ (local version) or of all $\mathrm{x}$ that are $\mathrm{F}$ (global version) are $\mathrm{F}$ simpliciter, but whether all or only some are $F$ in the same way, i.e. $F$ in virtue of being $G$ (where $G$ may pick out a plurality of properties). 
We can thus give the following revised duplication principles for the local and global notions of intrinsicality: ${ }^{31,32}$

- $\mathrm{Fx}_{\mathrm{INT}} \leftrightarrow \exists \mathrm{G}([\mathrm{Fx} \text { in virtue of } \mathrm{Gx}] \wedge \forall \mathrm{y}(\text { Dyx } \rightarrow[\text { Fy in virtue of } \mathrm{Gy}]))^{33}$

- $\mathrm{Fx}_{\mathrm{EXT}} \leftrightarrow \exists \mathrm{G}([\mathrm{Fx}$ in virtue of $\mathrm{Gx}] \wedge \exists \mathrm{y}(\mathrm{Dyx} \wedge \neg[\mathrm{Fy}$ in virtue of $\mathrm{Gy}]))$

- $\mathrm{F} \in\{\mathrm{I}\} \leftrightarrow \forall \mathrm{x} \forall \mathrm{G}([\mathrm{Fx}$ in virtue of $\mathrm{Gx}] \rightarrow \forall \mathrm{y}(\mathrm{Dyx} \rightarrow[$ Fy in virtue of $\mathrm{Gy}]))$

- $\mathrm{F} \in\{\mathrm{E}\} \leftrightarrow \exists \mathrm{x} \exists \mathrm{G}([\mathrm{Fx}$ in virtue of $\mathrm{Gx}] \wedge \exists \mathrm{y}(\mathrm{Dyx} \wedge \neg[\mathrm{Fy}$ in virtue of $\mathrm{Gy}]))$

These refined principles allow us to deal with necessary extrinsic properties since even though it is the case that duplicates never differ in terms of whether they instantiate an extrinsic necessary property, they do differ in terms of how they instantiate it. For instance, the necessary property formed by disjoining a pure extrinsic property with its complement, i.e. $F={ }_{\mathrm{df}}(\mathrm{G} \vee \neg \mathrm{G})$ turns out to be extrinsic since there will be an object $x$ that is $F$ in virtue of being $G$ as well as an

\footnotetext{
${ }^{3 \mathrm{I}}$ These principles only apply to non-empty and non-basic properties, i.e. properties that are had in virtue of other properties. Moreover, they are restricted to properties that are (in Kit Fine's terminology) 'wordly', i.e. those the instantiation of which depends on how things turn out, rather than 'unwordly', i.e. those that are instantiated regardless of how things turn out (cf. Fine: 2005 , ch. 9). This means that non-worldly necessary properties ('transcendent properties'), such as the property of being such that the number 2 exists, are excluded and that these principles only apply to those properties that depend on how the world turns out, to those properties that 'engage with the world', which in the case of extrinsic necessary properties amounts to depending on which disjunct of a necessary disjunction is realised.

${ }^{32}$ It should be noted that Lewis's account of duplication in terms of sharing perfectly natural properties and relations is not the only account that does not presuppose a notion of intrinsicality since duplication can be understood in terms of fundamental properties and relations. Accordingly, no commitment to naturalness is required.

DUPLICATE $^{*} \mathrm{x}$ and $\mathrm{y}$ are duplicates iff there is a bijection $\Gamma$ from the parts of $\mathrm{x}$ to the parts of $\mathrm{y}$, such that any fundamental property $\mathrm{F}$ is instantiated by any part $\mathrm{p}$ of $\mathrm{x}$ iff the image of $\mathrm{p}$ under $\Gamma$ is also $\mathrm{F}$, and such that any $\mathrm{n}$-ary fundamental relation $\mathrm{R}$ holds amongst parts $\mathrm{p}_{\mathrm{I}}$ $\ldots p_{n}$ of $x$ iff the images of $p_{I} \ldots p_{n}$ under $\Gamma$ also stand in $R$.
}

${ }^{33}$ If it should be possible for disjunctive properties to be grounded in other disjunctive properties (which depends on whether the 'in virtue of' relation relates disjunctive facts only to their ultimate grounds or also to non-ultimate grounds), then this condition needs to be modified. This is because counter-examples would arise insofar as one necessary extrinsic property, such as $\mathrm{F}=\mathrm{df}$ $\left(\mathrm{PE}_{\mathrm{I}} \vee \neg \mathrm{PE}_{\mathrm{I}} \vee \mathrm{PE}_{2}\right)$, could be grounded in another necessary extrinsic property, such as $\mathrm{G}=\mathrm{df}$ $\left(\mathrm{PE}_{\mathrm{I}} \vee \neg \mathrm{PE}_{\mathrm{I}}\right)$. In such a case, the property $\mathrm{F}$ would only be had extrinsically and not intrinsically by $\mathrm{x}$ even though there would be a property $\mathrm{G}$ had by $\mathrm{x}$ that is such that all duplicates of $\mathrm{x}$ are $\mathrm{F}$ in virtue of being $G$. That is, all duplicates of $x$ would be such as to share a common ground of Fness, which implies that the stated condition would misclassify Fness as being had intrinsically. This problem can be avoided by requiring that the common ground must itself be such that that in which it is grounded (if it is not fundamental) is a common ground.

- $\mathrm{Fx}_{\mathrm{INT}} \leftrightarrow \exists \mathrm{G}([\mathrm{Fx}$ in virtue of $\mathrm{Gx}] \wedge \forall \mathrm{y}(\mathrm{Dyx} \rightarrow([\mathrm{Fy}$ in virtue of $\mathrm{Gy}] \wedge \forall \mathrm{H}[\mathrm{Gx}$ in virtue of $\mathrm{Hx} \leftrightarrow$ Gy in virtue of $\mathrm{Hy}]))$ ) 
object that is $F$ in virtue of being $\neg \mathrm{G}$. Similarly, by assessing how properties are had, our refined duplication principles make room for mixed cases in which all duplicates are $F$ intrinsically, i.e. are $F$ in virtue of some intrinsic property $G$, and only some duplicates are $\mathrm{F}$ extrinsically, i.e. are $\mathrm{F}$ in virtue of some extrinsic property $\mathrm{H}$. This holds both for local mixed cases and for global mixed cases. For instance, when dealing with the disjunctive property $F={ }_{d f}(G \vee H)$, formed by disjoining an intrinsic property $\mathrm{G}$ with a pure extrinsic property $\mathrm{H}$, a local case arises in which there is an $\mathrm{x}$ that is $\mathrm{F}$ intrinsically in that there is a property, namely $G$, that is such that all duplicates of $x$ are $F$ in virtue of being $G$, whilst at the same time being $F$ extrinsically since $x$ is $F$ not only in virtue of being $G$ but also in virtue of being $\mathrm{H}$, where only some duplicates of $\mathrm{x}$ are $\mathrm{H}$. Likewise, the property $F={ }_{d f}(G \vee(G \wedge H))$ gives rise to global cases in which all $x$ are such that they (and all their duplicates) are $F$ in virtue of being $G$, yet only some $x$ are such they are $\mathrm{F}$ in virtue of being $\mathrm{H}$ and they will be such that not all of their duplicates are $\mathrm{H}$ and hence not $\mathrm{F}$ in virtue of being $\mathrm{H} .{ }^{34}$

SUPERVENIENCE: The reason we ran into problems with the supervenience principle is that supervenience is purely extensional in character. To remedy this problem we can replace the traditional supervenience principle by a grounding principle which states that all properties grounded in intrinsic properties are themselves intrinsic, while those grounded (at least partly) in extrinsic properties are also extrinsic. Since the notion of grounding is a hyperintensional notion, it does not suffer from the same defects as supervenience.

ClOSURE: We can also provide a proper statement of the closure conditions. Boolean combinations of properties that are always had intrinsically are always had intrinsically (if they are had at all). Similarly, Boolean combinations of properties that are always had purely extrinsically are always had purely extrinsically (if they are had at all).

When it comes to impure extrinsic properties, things get somewhat more complicated. The impure extrinsic properties come in two types, namely (i) those that are conjunctive in nature, i.e. ( $\wedge \mathrm{PE}$ ), and which are always had extrinsically though in an impure manner since they depend on how the object is both extrinsically as well as intrinsically and not purely on how it is extrinsically, and (ii) those that are disjunctive in nature, i.e. $(\mathrm{I} \vee \mathrm{PE})$, or $\left(\mathrm{I}_{\mathrm{I}} \wedge\left(\mathrm{I}_{2} \vee \mathrm{PE}\right)\right)$ or $\left(\mathrm{I}_{\mathrm{I}} \vee\right.$ $\left(\mathrm{I}_{2} \wedge \mathrm{PE}\right)$ ), and which are sometimes had intrinsically and sometimes (impurely or purely) extrinsically.

\footnotetext{
${ }^{34}$ There can also be mixed cases where all duplicates are $\mathrm{F}$ intrinsically and all duplicates are $\mathrm{F}$ extrinsically, as happens in the case of the following disjunctive property $((\mathrm{I} \vee \neg \mathrm{I}) \vee(\mathrm{PE} \vee \neg \mathrm{PE}))$. In such cases, all duplicates are $\mathrm{F}$ extrinsically though some are $\mathrm{F}$ extrinsically in virtue of some extrinsic property PE whilst others are F extrinsically in virtue of another extrinsic property $\neg \mathrm{PE}$.
} 
While the set of conjunctive impure extrinsics is not closed and while the set of disjunctive impure extrinsics is not closed either, the union of these sets, i.e. the set of impure extrinsic properties, is closed under Boolean operations. That is, properties formed out of conjunctive impure extrinsics can become disjunctive impure extrinsics as happens for example when we take the negation of $(\mathrm{I} \wedge \mathrm{PE})$, which can be had intrinsically if $\neg \mathrm{I}$ and can be had extrinsically if $\neg$ PE. Similarly, properties formed out of disjunctive impure extrinsics can become conjunctive impure extrinsics as happens for instance when we take the conjunction $(\mathrm{I} \vee \mathrm{PE})$ and ( $\neg \mathrm{I} \vee \neg \mathrm{PE}$ ), which is equivalent to the disjunctive property ( $(\mathrm{I} \wedge \neg \mathrm{PE}) \vee$ $(\neg \mathrm{I} \wedge \mathrm{PE}))$ and which is always had extrinsically and never intrinsically. Yet any combination of impure extrinsic properties is an impure extrinsic property (if it can be had at all). ${ }^{35}$ Accordingly, we can see that the set of impure extrinsic properties is closed under Boolean operations.

\subsection{Taking intrinsically/extrinsically as basic}

Both the combinatorial and the duplication approach are unable to provide an adequate account of the intrinsically/extrinsically distinction since they face serious problems with mixed properties and necessary/impossible properties. This is problematic not only because of the inability to adequately characterise the local notion of intrinsicality but also because a failure to capture the local notion threatens to undermine attempts to capture the global notion. This is because the local notion is prior to the global notion since the latter is specified in terms of the former. The intrinsicality status of a property is determined by how that property is had by different objects, namely whether it is always had intrinsically, always had extrinsically or sometimes had intrinsically and sometimes extrinsically. This means that identifying how a property is had by different objects is the primary issue. We can then specify what kind of property it is in terms of how it is had. Accordingly, in addition to being philosophically important in its own right, the local notion turns out to be crucial in explicating the global notion.

The reason for this is that we cannot go from the intrinsic/extrinsic distinction to the intrinsically/extrinsically distinction but only the other way around, making the latter distinction more basic than the former. This is because the fact that $\mathrm{F}$ is an extrinsic property underdetermines whether Fness is had intrinsically or extrinsically by x. All three cases, i.e. x's being F intrinsically, extrinsically, and intrinsically as well as extrinsically, are possible. All that follows from the fact that $\mathrm{F}$ is an extrinsic property is that it is not always had only intrinsically,

\footnotetext{
${ }^{35}$ Here we are again crucially relying on hyperintensional criteria for property instantiations since impure extrinsic properties can be combined in such a way that the combination can be extensionally equivalent to an intrinsic property as happens with the disjunction $((\mathrm{I} \wedge \mathrm{PE}) \vee(\mathrm{I}$ $\wedge \neg \mathrm{PE})$ ). Though extensionally equivalent to intrinsic properties, these properties can be distinguished therefrom by our hyperintensional account, allowing us to accept the closure condition even for the impure extrinsic properties.
} 
thereby leaving several options open.

Yet, when we take the intrinsically/extrinsically distinction as basic, we can specify all the different ways in which a property can be had and all the different kinds of intrinsicality statuses that a property can have: ${ }^{36}$

- $\mathrm{F}$ is neither intrinsic nor extrinsic $=\neg \exists \mathrm{x}\left(\mathrm{Fx}_{\mathrm{INT}}\right) \wedge \neg \exists \mathrm{x}\left(\mathrm{Fx}_{\mathrm{EXT}}\right)$

- $\mathrm{F}$ is intrinsic $=\forall \mathrm{x}\left(\mathrm{Fx} \rightarrow \mathrm{Fx}_{\mathrm{INT}}\right) \wedge \neg \exists \mathrm{x}\left(\mathrm{Fx}_{\mathrm{EXT}}\right)$

- $\mathrm{F}$ is extrinsic $=\exists \mathrm{x}\left(\mathrm{Fx}_{\mathrm{EXT}}\right)$

- $\mathrm{F}$ is exclusively extrinsic $=\forall \mathrm{x}\left(\mathrm{Fx} \rightarrow \mathrm{Fx}_{\mathrm{EXT}}\right) \wedge \neg \exists \mathrm{x}\left(\mathrm{Fx}_{\mathrm{INT}}\right)$

- $\mathrm{F}$ is hybrid $=\exists \mathrm{x}\left(\mathrm{Fx}_{\mathrm{INT}} \wedge \mathrm{Fx}_{\mathrm{EXT}}\right)$

- $\mathrm{F}$ is anti-hybrid $=\exists \mathrm{x}\left(\mathrm{Fx}_{\mathrm{INT}}\right) \wedge \exists \mathrm{x}\left(\mathrm{Fx}_{\mathrm{EXT}}\right) \wedge \neg \exists \mathrm{x}\left(\mathrm{Fx}_{\mathrm{INT}} \wedge \mathrm{Fx}_{\mathrm{EXT}}\right)$

If $\mathrm{F}$ is an impossible property, then it is never had at all and it is a fortiori never had either intrinsically or extrinsically. Such a property is consequently neither intrinsic nor extrinsic.

If $\mathrm{F}$ is always had only intrinsically, then $\mathrm{F}$ is an intrinsic property. It is a property that is wholly and solely had in virtue of a how a thing itself is. ${ }^{37}$

If $\mathrm{F}$ is sometimes had extrinsically, then it is an extrinsic property. It is a property that is not always wholly and solely had in virtue of how a thing itself is, but at least sometimes had at least partly in virtue of how a thing is extrinsically, of how it is related to other things.

Extrinsic properties can be distinguished into (i) exclusively extrinsic properties that are never had intrinsically, which can in turn be distinguished into (ia) those that are never partly had in virtue of how a thing itself is (the pure cases), and (ib) those that are conjunctive in nature and are at least sometimes had partly in virtue of how a thing itself is (the impure cases), (ii) hybrid properties that are disjunctive in nature and that can be had both intrinsically and extrinsically, which can in turn be distinguished into (iia) those that are always had both intrinsically and extrinsically (global hybrid), and (iib) those that are only sometimes had both intrinsically and extrinsically (local hybrid), and (iii) anti-hybrid properties that

\footnotetext{
${ }^{36}$ The definitions of intrinsic and extrinsic properties only apply to non-empty properties (cf. section 4).

${ }^{37}$ The idea that a property $\mathrm{F}$ is intrinsic iff it is always had intrinsically (and never extrinsically) has been questioned on the basis that there can be disjunctive properties with impossible disjuncts that are always had intrinsically but that do not seem to be intrinsic properties (cf. Weatherson: 2006, section I.2). Properties such as $(\mathrm{I} \vee(\mathrm{PE} \wedge \neg \mathrm{PE}))$ can be had in different ways, namely by being ( $\wedge$ PE) or by being $(\mathrm{I} \wedge \neg \mathrm{PE}$ ). Extrinsic factors determine the way in which they are had, which implies that these properties are correctly classified by our account as being extrinsic. If there should be basic impossible properties, i.e. impossible properties that do not result from impossible combinations of properties, then such basic impossible disjuncts do not affect the ways in which a disjunctive property can be had. Given our hyperintensional criterion of property individuation, this implies that they do not affect the identity of a property (cf. section 5.I). As a result, disjunctive properties with basic impossible disjuncts are correctly classified by our account as being intrinsic.
} 
are disjunctive in nature and are either had intrinsically or extrinsically but that can never be had in both ways.

\subsection{Fundamental properties and relations}

An adequate analysis of intrinsicality needs to specify that in virtue of which properties are had intrinsically or extrinsically without bringing in any intrinsicality notions. This can be done by appealing to the notion of 'fundamentality', as well as to the distinction between relations and properties. The notion of a fundamental property/relation can be defined in terms of the 'in virtue of' locution. A property $\mathrm{F}$ is a fundamental property iff it is the case that for all $\mathrm{x}(\mathrm{i})$ there is no property $G$ such that $x$ is $G$ and $x$ is $F$ (at least partly) in virtue of being $G$, and (ii) there is no relation $R$ such that x's being $F$ is (at least partly) grounded in (iia) x's being R-related to some y or (iib) x's failing to be R-related to some y. A relation $\mathrm{R}$ is a fundamental relation iff it is the case that for all $\mathrm{x}(\mathrm{i})$ there is no property G such that $x$ is $G$ and $x$ stands in $R$ (at least partly) in virtue of being $G$, and (ii) there is no relation $\mathrm{R}^{*}$ such that x's standing in $\mathrm{R}$ is (at least partly) grounded in (iia) x's being $\mathrm{R}^{*}$-related to some y or (iib) x's failing to be $\mathrm{R}^{*}$-related to some $\mathrm{y}$. We can then distinguish whether properties are had intrinsically or extrinsically in terms of how they are grounded in fundamental properties and relations. The resulting theory is accordingly developed by means of the fundamental $\mathrm{v}$. non-fundamental distinction and the relation v. property distinction. ${ }^{38}$

A property $\mathrm{F}$ is had only intrinsically and not extrinsically by $\mathrm{x}$ iff $\mathrm{x}$ is $\mathrm{F}$ and either (i) $\mathrm{F}$ is a fundamental property, or (ii) $\mathrm{F}$ is a nonfundamental property that is either solely grounded in (iia) fundamental properties of $x$, or in (iib) fundamental properties and fundamental relations holding amongst x's (proper and improper) parts, or in (iic) fundamental relations holding amongst x's (proper and improper) parts, or in (iid) any combination of (iia)-(iic). ${ }^{39}$

A property $\mathrm{F}$ is had both intrinsically and extrinsically by $\mathrm{x}$ iff $\mathrm{F}$ is a non-fundamental property had by $\mathrm{x}$ that is wholly but not solely

\footnotetext{
${ }^{38}$ Humberstone argues that the intrinsic/extrinsic distinction applies to properties, while the relational/non-relational distinction applies to concepts (cf. Humberstone: 1996). (This is endorsed by, amongst others, Weatherson and Langton.) However, the reason Humberstone gives for accepting this bifurcation disappears once we accept a hyperintensional understanding of properties. Since we have to draw hyperintensional distinctions amongst properties and not only amongst concepts, we can see that both the intrinsic/extrinsic distinction and the relational/nonrelational distinction apply to properties and that we can accordingly use the latter distinction in analysing the former.

${ }^{39}$ Parthood is to be construed broadly such that, for instance, the members of a set classify as its parts (cf. Fine: 2010). This allows the proposed theory of intrinsicality to be applicable to entities other than material objects such as sets, structural universals, facts and so on.
} 
grounded in (ia) fundamental properties of $\mathrm{x}$, or in (ib) fundamental properties and fundamental relations holding amongst x's (proper and improper) parts, or in (ic) fundamental relations holding amongst x's (proper and improper) parts, and that is not solely grounded in (id) any combination of (ia)-(ic).

This account presupposes that all fundamental properties are always had only intrinsically and are therefore intrinsic properties. This means that there are no fundamental extrinsic properties and that at the fundamental level of the property hierarchy we only have intrinsic relations and intrinsic properties. The reason for this is that properties that are had extrinsically are all derivative and to be found at non-fundamental levels. They are derivative because all extrinsic properties are relational in character and properties that are relational in character derive from the obtaining or non-obtaining of relations and are consequently non-fundamental. ${ }^{40}$ Alternatively, if a property is fundamental, then it cannot be had in virtue of any relations and if is not had in virtue of any relations, then it is had intrinsically.

One reason for believing that positive relational properties are derivative is that we would otherwise run into problems in accounting for the fact that different relational properties had by objects that are mereologically disjoint are coordinated and not separately recombinable. For example, if $\mathrm{a}$ and $\mathrm{b}$ stand in a symmetric relation $\mathrm{R}$, i.e. if Rab, then a has the property of being $\mathrm{R}$-related to $\mathrm{b}$, i.e. $\lambda x[R x b] a$, and $b$ has the property of being R-related to a, i.e. $\lambda x[R x a] b$. By taking relations to be more fundamental than relational properties we can explain the fact that if we have $\lambda \mathrm{x}[\mathrm{Rxb}] \mathrm{a}$, then we also have $\lambda \mathrm{x}[\mathrm{Rxa}] \mathrm{b}$. If both properties derive from the same relation, then the fact that the relation obtains suffices for the fact that these two properties are instantiated. If, however, the relational properties are considered to be fundamental, then it becomes difficult to explain why they cannot be separately recombined and why the fact that one of them is instantiated requires that the other is instantiated as well..$^{41,42}$

Specifying when a property $\mathrm{F}$ is had extrinsically is more tricky. Given that there are mixed cases, we cannot straightforwardly define it negatively in the sense that $\mathrm{F}$ is had extrinsically when it is not had intrinsically since this does not adequately account for cases in which it is had both intrinsically and extrinsically.

\footnotetext{
${ }^{40}$ Positive relational properties result from $\mathrm{x}$ standing in some relation $\mathrm{R}$, whilst negative relational properties result from $\mathrm{x}$ failing to stand in some relation $\mathrm{R}$. In each case the relational property is derivative.

${ }^{4 \mathrm{I}}$ In the case of non-symmetric relations, we need to explain not only why $\lambda \mathrm{x}[\mathrm{Rxb}] \mathrm{a}$ and $\lambda \mathrm{x}\left[\mathrm{R}^{-\mathrm{I}} \mathrm{xa}\right] \mathrm{b}$ are not separately recombinable, but also why $\mathrm{Rab}$ and its converse $\mathrm{R}^{-\mathrm{I}}$ ba are not separately recombinable (cf. Williamson: 1985, Fine: 2000, Dorr: 2004, van Inwagen: forthcoming).

${ }^{42}$ In the case of internal relations, i.e. relations that are reducible to the intrinsic properties of their relata, both the relational property and the corresponding relation are reducible and derivative.
} 
Nor can we positively specify wherein the extrinsic possession of properties is grounded, since we run into difficulties when it comes to negative relational properties.

As long as we are dealing with positive relational properties, we can give an analysis of what it takes for $\mathrm{x}$ to be $\mathrm{F}$ extrinsically in terms of conditions referring to properties of and/or relations to objects that are mereologically distinct from $\mathrm{x}$. Problems arise, however, when dealing with certain negative relational properties, such as the property of being lonely. In particular, the problem is to account for cases where there is no $y$ distinct from $\mathrm{x}$, such that $\mathrm{x}$ bears $\mathrm{R}$ to $\mathrm{y}$, making it the case that $\mathrm{x}$ has the property of not being R-related to any $\mathrm{y}$. It is not clear what these properties are grounded in. This problem is closely related to that of finding grounds for negative existentials and totality facts. We will not attempt to give a solution to this problem here. ${ }^{43}$

\footnotetext{
${ }^{43} \mathrm{~A}$ common way of trying to deal with negative relational properties such as lonelines consists in providing analyses that simultaneously assess $\mathrm{F}$ and $\neg \mathrm{F}$. In that way one can ensure that, even though $\mathrm{F}$ might not be grounded in how the rest of the world is given that $\mathrm{F}$ involves loneliness, $\neg \mathrm{F}$ will be at least partially grounded in how the rest of the world is since it will involve accompaniment. For instance, Gideon Rosen has recently proposed an account of intrinsicality that takes this form (Rosen: 2010, p. I I 2):

" $\mathrm{F}$ is an intrinsic property iff, as a matter of necessity, for all $\mathrm{x}$ :

$$
\begin{aligned}
& \text { If } \mathrm{x} \text { is } \mathrm{F} \text { in virtue of } \phi(\mathrm{y})-\text { where } \phi(\mathrm{y}) \text { is a fact containing } \mathrm{y} \text { as a constituent - then } \\
& \mathrm{y} \text { is part of } \mathrm{x} \text {; and } \\
& \text { If } \mathrm{x} \text { is not-F in virtue of } \phi(\mathrm{y}) \text {, then } \mathrm{y} \text { is part of } \mathrm{x} \text {. }
\end{aligned}
$$
}

(The last clause ensures that loneliness - the property a thing has when there are no things distinct from it - is not deemed an intrinsic property.)"

These kinds of accounts, however, are subject to counter-examples since the property $\mathrm{F}={ }_{\mathrm{df}}((\mathrm{I}$ $\vee \neg I) \vee L$ ), where I is an arbitrary intrinsic property and where $L$ is the property of being lonely, is extrinsic since how it is instantiated depends on whether an object is lonely. (If something is $\mathrm{I} \wedge \mathrm{L}$, then it is $\mathrm{F}$ partly in virtue of being $\mathrm{L}$ and any property that is partly grounded in how a thing is extrinsically is an extrinsic property.) Yet, this property satisfies both conditions. It satisfies condition (I) since Fx can be had in virtue of I or $\neg \mathrm{I}$ or L. I and $\neg \mathrm{I}$ are intrinsic and if it is had in virtue of $\mathrm{L}$, then it also satisfies condition (I) since loneliness is neither grounded in any properties of the parts of a lonely object, nor in any properties of the parts of objects disjoint from a lonely object since ex hypothesi no such objects exist (this is after all why Rosen imposes condition (2)). It also satisfies condition (2) since the complement of the property, i.e. not-F, is $((\mathrm{I} \wedge \neg \mathrm{I}) \wedge \neg \mathrm{L})$. Since this is an impossible property that is never had, it follows that condition (2) is trivially satisfied. Put differently, since the negation of F involves an impossible property, this property will never be instantiated and its instantiation will consequently never be partially grounded in the way the rest of the world is.

Moreover, all these accounts have the problematic consequence that the set of intrinsic properties will be fully closed, i.e. $\mathrm{F}$ is intrinsic iff $\neg \mathrm{F}$ is intrinsic. Accordingly, they make those impossible properties that are complements of necessary intrinsic properties also intrinsic, and thereby ensure that certain properties are classified as intrinsic even though there is nothing that has them intrinsically. 
Fortunately though, we do not need to give an account of negative existentials for the purposes of this paper since we can identify conditions that allow us to specify all those cases where Fness is had extrinsically by $\mathrm{x}$ without specifying in each instance wherein the possession of the property is grounded. We can do this by appealing to the distinction between being wholly and being solely grounded.

In particular, we can say that a non-fundamental property $\mathrm{F}$ is had extrinsically by $\mathrm{x}$ in all those cases in which it is not solely grounded in fundamental properties of and/or fundamental relations holding amongst x's proper and improper parts. Not being solely grounded in fundamental properties of and/or fundamental relations holding amongst x's proper and improper parts is compatible with x's Fness being both wholly grounded in fundamental properties of and/or fundamental relations holding amongst x's proper and improper parts as well as wholly/partly grounded in something else, thereby making room for mixed cases. Accordingly, we can negatively specify when a property is had extrinsically, namely when it is not solely grounded in fundamental properties and/or relations, without mistakenly ruling out mixed cases.

A property $\mathrm{F}$ is had extrinsically by $\mathrm{x}$ iff $\mathrm{F}$ is a non-fundamental property had by $\mathrm{x}$ that is not solely grounded in (ia) fundamental properties of $\mathrm{x}$, and that is not solely grounded in (ib) fundamental properties and fundamental relations holding amongst x's (proper and improper) parts, and that is not solely grounded in (ic) fundamental relations holding amongst x's (proper and improper) parts, and that is not solely grounded in (id) any combination of (ia)-(ic).

We can then give more fine-grained distinctions of how a property can be had extrinsically by $\mathrm{x}$, namely whether it is

(i) wholly but not solely grounded in fundamental properties of and/or relations holding amongst x's proper and improper parts (mixed cases),

(ii) partly but not wholly grounded in fundamental properties of and/or relations holding amongst x's proper and improper parts (impure cases), or

(iii) not at all grounded in fundamental properties of and/or relations holding amongst x's proper and improper parts (pure cases).

While properties of type (i) are disjunctive and can be had wholly in virtue of the intrinsic disjunct as well as wholly in virtue of the extrinsic disjunct, those of type (ii) are conjunctive and are had partly in virtue of the intrinsic conjunct and partly in virtue of the extrinsic conjunct. 


\subsection{Fundamentality v. naturalness}

There are three further advantages of working with 'in virtue of' claims and the corresponding notion of 'fundamentality' rather than with 'naturalness'.

First, those appealing to naturalness face the problem of ruling out perfectly natural extrinsic properties (cf. Yablo: I999, p. 480). Lewis accepts that there are perfectly natural relations but excludes the possibility of perfectly natural relational properties. Yet, it is not clear why a relational property should necessarily be less natural than the relation from which it derives. Those appealing to fundamentality do not have an analogous problem since they can explain why a relational property is less fundamental than the relation. This simply follows from the fact that the property is derivative on the relation. Relational properties turn out to be non-fundamental since they are grounded in the relation, i.e. $\mathrm{x}$ has a relational property $\mathrm{F}$ in virtue of standing in a relation $\mathrm{R}$, making the relation more fundamental than the relational property (cf. section 5.4).

Second, we noted in section I. 3 that the duplication approach requires there to be perfectly natural properties, which would be a problematic commitment if the property hierarchy could turn out to lack a lower bound. It might be objected that the account of intrinsicality in terms of 'in virtue of' claims also presupposes there to be a lower bound, i.e. a fundamental level in the property hierarchy. ${ }^{44}$ While this is true, this presupposition can be explained in terms of the very notion of grounding. For an account as to why we are justified in expecting the grounding relation to give rise to a lower-bounded partial ordering, consisting of chains of conditions that terminate in ultimate grounds cf. Fine: $200 \mathrm{I}$, p. 27 footnote 38 and Schaffer: manuscript, p. 23. Yet, there is nothing in the notion of naturalness that implies that there are perfectly natural properties. This means that both accounts have similar commitments, but only the in virtue of' account has the relevant resources to defend its commitments.

In this context, it is worth pointing out that the refined duplication principles that were developed in section 5.2 do not explicitly appeal to the notion of a fundamental property/relation and accordingly might be applicable in cases in which the property hierarchy should turn out to lack a lower bound. Whilst this feature is compromised by the characterisation of duplication that we provided since we appealed to the notion of a fundamental property/relation, it is possible to provide an alternative account of duplication that does not have this commitment. This can be done, for instance, by appealing to chains of more and more fundamental properties of the same type. In particular, a monadic property $\mathrm{F}$ is a member of a fundamentality chain if all its grounds are monadic properties, i.e.

${ }^{44} \mathrm{It}$ is important to note that what is at issue is whether the property hierarchy lacks a lowerbound, i.e. whether there are chains of properties that are more and more fundamental but do not bottom out in fundamental properties. The account developed in this paper is compatible with the possibility of the mereological hierarchy lacking a lower bound, i.e. with the possibility of there being gunk. 
any fact that grounds x's being $\mathrm{F}$ that has $\mathrm{x}$ as a constituent is a fact to the effect that $\mathrm{x}$ instantiates a monadic property. Likewise, an $\mathrm{n}$-ary relation $\mathrm{R}$ is a member of a fundamentality chain if all its grounds are n-ary relations, i.e. any fact that grounds x's standing in $\mathrm{R}$ that has $\mathrm{x}$ as a constituent is a fact to the effect that $\mathrm{x}$ stands in an n-ary relation. This then allows us to give an alternative account of duplication that is compatible with the property hierarchy lacking a lower bound:

DUPLICATE $^{* *} \mathrm{x}$ and $\mathrm{y}$ are duplicates iff there is a bijection $\Gamma$ from the parts of $\mathrm{x}$ to the parts of $y$, such that any property $F$ that is a member of a fundamentality chain is instantiated by any part $\mathrm{p}$ of $\mathrm{x}$ iff the image of $\mathrm{p}$ under $\Gamma$ is also $\mathrm{F}$, and such that any $n$-ary relation $R$ that is a member of a fundamentality chain holds amongst parts $\mathrm{p}_{\mathrm{I}} \ldots \mathrm{p}_{\mathrm{n}}$ of $\mathrm{x}$ iff the images of $\mathrm{p}_{\mathrm{I}} \ldots \mathrm{p}_{\mathrm{n}}$ under $\Gamma$ also stand in $\mathrm{R}$.

Third, the naturalness approach faces difficulties when it comes to dealing with non-qualitative properties and relations since it is unclear whether it even makes sense to apply the notion of naturalness to such properties and relations. For instance, if perfect naturalness is specified in terms of the properties over which second-order quantifiers range in the final theory $\mathrm{T}$, then naturalness will not be applicable to non-qualitative properties since science does not deal with such properties. Similarly, if naturalness is concerned with the privileged set of properties that give rise to genuine similarity, then again it seems that the notion of naturalness is not applicable to non-qualitative properties and relations since similarity is not understood in terms of these properties and relations. We can, however, make sense of fundamental non-qualitative properties and fundamental non-qualitative relations and thereby employ the proposed analysis for dealing with all properties and relations, rather than being at best able to provide a restricted analysis. Thus, the fact that the proposed account of intrinsicality is framed in terms of 'in virtue of' claims rather than in terms of 'naturalness' claims allows us to apply it to haecceistic or non-qualitative properties (cf. Bader: manuscript-b).

\section{Conclusion}

Thus, we have seen that duplication and combinatorial accounts are inadequate and that appealing to naturalness is to no avail. These accounts mistakenly rule out cases in which a property is possessed both intrinsically and extrinsically, which occurs for instance if an object is both intrinsically and extrinsically valuable. Extensional accounts cannot distinguish being $\mathrm{F}$ only intrinsically from being $\mathrm{F}$ both intrinsically and extrinsically. Put differently, they cannot distinguish being $\mathrm{F}$ solely in virtue of how a thing itself is from being $\mathrm{F}$ wholly but not solely in virtue of how a thing itself is. In addition, these accounts cannot adequately deal with extra-world sources of extrinsicness. This leads them to 
misclassify certain modal properties when these are given a counterpart-theoretic analysis. Finally, these accounts have difficulty in dealing with necessary as well as impossible properties and are unable to retain the idea that the set of intrinsic properties and the set of pure extrinsic properties are closed under Boolean operations.

The underlying problem is that duplication and combinatorial principles are framed in terms of an extensional understanding of how properties are instantiated and individuated. They only look at whether a property is had but not at how it is had, thereby ignoring important hyperintensional differences in the ways in which properties can be had. Instead of providing extensional accounts supplemented by naturalness, we need a more fine-grained account of how properties are instantiated and individuated, allowing us to specify different ways in which properties can be had.

In particular, we need an account based on 'in virtue of' claims that allows us to specify different ways in which a property can be had. ${ }^{45}$ This then makes room for refined versions of duplication, supervenience and closure principles that characterise intrinsicality. The resulting theory will take the intrinsically/extrinsically distinction as more basic than the intrinsic/extrinsic distinction and will define the latter in terms of the former. By taking the local notion to be more basic than the global notion we can account for mixed cases as well as give a principled reason as to why downward closure fails, i.e. why impossible properties are neither intrinsic nor extrinsic. Whether a property is had intrinsically and/or extrinsically is then defined in terms of the fundamental properties and/or relations in virtue of which it is had.

LOCAL A property $\mathrm{F}$ is had intrinsically by $\mathrm{x}$ iff $\mathrm{x}$ is $\mathrm{F}$ wholly in virtue of how it itself is or $\mathrm{F}$ is a fundamental property had by $\mathrm{x}$.

GLOBAL A property $\mathrm{F}$ is an intrinsic property iff $\mathrm{F}$ is always had solely in virtue of how a thing itself is or $\mathrm{F}$ is a fundamental property.

How an object itself is can then be understood in terms of the fundamental properties of and the fundamental relations holding amongst the proper and improper parts of that object. In this way, we are able to give a complete account of intrinsicality that allows for mixed cases, is able to deal with extra-world sources of extrinsicness, can adequately classify impossible and necessary properties, and applies to qualitative as well as non-qualitative properties and relations. ${ }^{46}$

\footnotetext{
${ }^{45}$ In addition, we need to recognise the hyperintensional distinction between the source of a property and the condition of a property in order to develop a relativised notion of intrinsicality, which is for instance required if we are to make sense of conditional intrinsic value (cf. Bader: manuscript-c).

${ }^{46}$ For helpful comments I am grateful to Lauren Ashwell, Joachim Aufderheide, Derek Ball, John Collins, Katherine Hawley, and the editors of this journal. Thanks are also due to audiences at St Andrews, the TIF XII at Girona, the APA Pacific Division meeting in San Francisco, and the metaphysics reading group at Rutgers.
} 


\section{References}

[I] Bader, R. M. Admissible Factorisations and Hyperintensional Independence. Manuscript-a.

[2] Bader, R. M. Duplication Principles for Non-Qualitative Properties. Manuscript-b.

[3] BADER, R. M. Relativised Intrinsicality. Manuscript-c.

[4] Denby, D. The Distinction between Intrinsic and Extrinsic Properties. Mind IIS, 457 (2006), I-I7.

[5] Dorr, C. Non-Symmetric Relations. Oxford Studies in Metaphysics I (2004), I 55-I92.

[6] Dunn, J. M. Relevant Predication 2: Intrinsic Properties and Internal Relations. Philosophical Studies 60 (I990), I77-206.

[7] Eddon, M. Intrinsicality and Hyperintensionality. Philosophy and Phenomenological Research 82, 2 (201 I), 3 I 4-336.

[8] Fine, K. Neutral Relations. The Philosophical Review I09, I (2000), I-33.

[9] Fine, K. The Question of Realism. Philosopher's Imprint I, I (200 I), I-30.

[ıо] Fine, K. Modality and Tense: Philosophical Papers. Oxford University Press, 2005 .

[i i] Fine, K. Towards a Theory of Part. The Journal of Philosophy I07, I I (20 I o), 559-589.

[i 2] Humberstone, I. L. Intrinsic/Extrinsic. Synthese I08 (I996), 205-267.

[13] KIm, J. Psychophysical Supervenience. Philosophical Studies 4I (I982), $5 \mathrm{I}-7 \mathrm{O}$.

[i4] Langton, R., and Lewis, D. Defining 'Intrinsic'. Philosophy and Phenomenological Research 58, 2 (I998), 333-345.

[I 5] LewIs, D. New Work for a Theory of Universals. Australasian Journal of Philosophy 6I, 4 (I983a), 343-377.

[16] Lewis, D. Extrinsic Properties. Philosophical Studies 44 (1983b), I97-200.

[I7] Lewis, D. On the Plurality of Worlds. Blackwell Publishers, I 986.

[I 8] LewIs, D. Redefining 'Intrinsic'. Philosophy and Phenomenological Research 63, 2 (200I), 38I-398. 
[i 9] Marshall, D. Can 'Intrinsic' Be Defined Using Only Broadly Logical Notions? Philosophy and Phenomenological Research 78, 3 (2009), 646-672.

[20] Parsons, J. Theories of Persistence. PhD thesis, Australian National University, 200I.

[2 I] Regan, D. How to Be a Moorean. Ethics II3 (2003), 65 I-677.

[22] Rosen, G. Metaphysical Dependence: Grounding and Reduction. In Modality: Metaphysics, Logic, and Epistemology, B. Hale and A. Hoffmann, Eds. Oxford University Press, 2010, pp. 109-135.

[23] Schaffer, J. On What Grounds What. Manuscript.

[24] Sider, T. Intrinsic Properties. Philosophical Studies 83 (I996), I-27.

[25] Sider, T. Maximality and Intrinsic Properties. Philosophy and Phenomenological Research 63, 2 (2001), 357-364.

[26] van Inwagen, P. Names for Relations. Philosophical Perspectives (forthcoming).

[27] Weatherson, B. Intrinsic Properties and Combinatorial Principles. Philosophy and Phenomenological Research 63, 2 (2001), 365-380.

[28] Weatherson, B. Intrinsic vs. Extrinsic Properties. Stanford Encylopedia of Philosophy (2006).

[29] Williamson, T. Converse Relations. The Philosophical Review 94, 2 ( 1985 ), 249-262.

[30] Witmer, D., Butchard, W., and Trogdon, K. Intrinsicality without Naturalness. Philosophy and Phenomenological Research 70, 2 (2005), 326-350.

[3 I] Yablo, S. Intrinsicness. Philosophical Topics 26, I/2 (I999), 479-505.

[32] Zimmerman, D. Immanent Causation. Philosophical Perspectives II (I997), $433-47$ I 